\title{
ON A COMPLETE SYSTEM OF INVARIANTS OF TWO TRIANGLES*
}

\author{
BY \\ DAVID D. LEIB \\ Introductory.
}

The simpler invariants of two triangles, arising from the connex set up by them when regarded as two cubic curves of the third order and of the third class, respectively, were discussed by Dr. HuN in vol. 5 of these Transactions, pages 39-55. The present paper exhibits $a$ complete system by means of which all invariant relations can be conveniently expressed, and is largely the result of an attempt to solve a problem proposed by Professor MorLey : What is the invariant relation between two triangles so that a conic may be drawn on the vertices of one triangle and touching the sides of the other? In this and similar problems, the one triangle was invariably taken as the reference triangle, and the invariant relation consequently expressed in terms of the coefficients of the other, a simplification which we shall use throughout the article. In this way the selection of the fundamental system was largely a matter of experiment in the first place, the object being to select such as would enable one to write down invariant relations in the most simple form, and at the same time to have these fundamental invariants easily interpreted geometrically. In the body of this paper we shall follow the logical order, however, of giving the system first and then proving its completeness.

\section{§1. The fundamental system.}

If we take one triangle as a 3 -line given by the symbolic equation

and the other as a 3-point

$$
(\alpha x)(\beta x)(\gamma x)=0,
$$

$$
(a \xi)(b \xi)(c \xi)=0 \text {, }
$$

where $(\alpha x)=\alpha_{1} x_{1}+\alpha_{2} x_{2}+\alpha_{3} x_{3}$, etc., the constants $a_{i}, b_{i}, c_{i}, \alpha_{i}, \beta_{i}, \gamma_{i}$ form the domain of rationality for the invariants. The six fundamental invariants $\Delta_{1}, D_{1}, I_{1}, D_{2}, I_{2}$ and $I_{3}$, expressed rationally and integrally in this domain are given on page 128 of The Johns Hopkins University Circular, July, 1908. Of these, $\Delta_{1}$ and $D_{1}$ are the determinants of the 3-point and 3-line respectively.

\footnotetext{
* Presented to the Society, December 31, 1908.
} 
That is

$$
\Delta_{1}=\mid \begin{array}{lll}
\alpha_{1} & \beta_{1} & \gamma_{1} \\
\alpha_{2} & \beta_{2} & \gamma_{2} \\
\alpha_{3} & \beta_{3} & \gamma_{3}
\end{array}
$$

Following Dr. Hus's notation for indicating the collective degree in Roman and Greek letters respectively, the invariants are represented as follows: $D_{1}(3,0) ; \Delta_{1}(0,3) ; I_{1}(3,3) ; I_{2}(6,6) ; D_{2}(6,6) ; I_{3}(9,9)$. From these can be formed four independent absolute invariants of zero degree,

$$
\begin{array}{lll}
I_{2} & \frac{I_{1}}{D_{2}}, & \frac{I_{1}^{2}}{\Delta_{1} \bar{D}_{1}},
\end{array} \quad \frac{I_{1} I_{2}}{I_{3}},
$$

and in terms of these four can be expressed rationally every other rational invariant of zero degree in both sets of coefficients. This is a minimum reduced system, since two triangles have projectively four absolute invariants.

If now the 3-line be taken as the reference triangle, $\Delta_{1}$ assumes the value unity and the other fundamental invariants become homogeneous, rational integral functions of the coefficients of the 3-point, as follows :

$$
\begin{gathered}
D_{1}=\left|\begin{array}{lll}
a_{1} & b_{1} & c_{1} \\
a_{2} & b_{2} & c_{2} \\
a_{3} & b_{3} & c_{3}
\end{array}\right|, \\
I_{1}=a_{1} b_{2} c_{3}+a_{3} b_{11} c_{2}+a_{2} b_{3} c_{1}+a_{1} b_{3} c_{2}+a_{2} b_{1} c_{3}+a_{3} b_{2} c_{1}, \\
D_{2}=\left|\begin{array}{lll}
a_{2} a_{3} & b_{2} b_{3} & c_{2} c_{3} \\
a_{3} a_{1} & b_{3} b_{1} & c_{3} c_{1} \\
a_{1} a_{2} & b_{1} b_{2} & c_{1} c_{2}
\end{array}\right|, \\
I_{2}=a_{2} a_{3} b_{3} b_{1} c_{1} c_{2}+a_{1} a_{2} b_{2} b_{3} c_{3} c_{1}+a_{3} a_{1} b_{1} b_{2} c_{2} c_{3}+a_{2} a_{3} b_{1} b_{2} c_{3} c_{1}+a_{1} a_{2} b_{3} b_{1} c_{2} c_{3}+a_{3} a_{1} b_{2} b_{3} c_{1} c_{2}, \\
I_{3}=a_{1} a_{2} a_{3} b_{1} b_{2} b_{3} c_{1} c_{2} c_{3} .
\end{gathered}
$$

It is in this simplified form that the invariants are most available, and it is a matter of very little difficulty to introduce $\Delta_{1}$ into any invariant form so as to render it homogeneous and of the proper degree in the Greek letters. The invariants $D_{1}$ and $D_{2}$ are skew, as is evident from their form.

\section{§2. Proof of the completeness of the system.}

Take the 3-line as reference triangle and call it $T_{1}$. The 3-point, $(a \xi)(b \xi)$ (c $\xi)=0$, we shall call $T_{2}$. If $T_{1}$ is taken as $(\alpha x)(\beta x)(\gamma x)=0$, we shall define a rational simultaneous invariant of $T_{1}$ and $T_{2}$ as a rational integral func- 
tion of the coefficients, which has the invariant property and is unaltered by any permutation of the three lines or of the three points; that is, by interchanges of $\alpha_{i}, \beta_{i}, \gamma_{i}$ or of $a_{i}, b_{i}, c_{i}$. When $T_{1}$ is taken as the reference triangle, all rational simultaneous invariants (except $\Delta_{1}$, which becomes unity) of $T_{1}$ and $T_{2}$ are homogeneous, rational, integral functions of $a_{i}, b_{i}, c_{i}$, which are unaltered by all permutations of the letters and of the subscripts.

The converse is equally true, and any homogeneous, rational, integral function of $a_{\imath}, b_{\imath}, c_{\imath}$, which is left unaltered by permutation of the letters or of the subscripts, is equivalent to an explicit rational integral function homogeneous in both the Roman and the Greek letters, which has the invariant property under all linear transformations of the plane. Hence the most general form of such an invariant is

where

$$
I=\sum a_{1}^{i_{1}} a_{2}^{i_{2}} a_{3}^{i_{3}} b_{1}^{j_{1}} b_{2}^{j_{2}} b_{3}^{j_{3}} c_{1}^{k_{1}} c_{2}^{k_{9}} c_{3}^{k_{3}}
$$

$$
\begin{array}{ll}
i_{1}+i_{2}+i_{3}=n, & i_{1}+j_{1}+k_{1}=n, \\
j_{1}+j_{2}+j_{3}=n, & i_{2}+j_{2}+k_{2}=n, \\
k_{1}+k_{2}+k_{3}=n, & i_{3}+j_{3}+k_{3}=n .
\end{array}
$$

We next prove the lemma: Every individual term of $I$, where $I$ is of the $n$th degree in the coefficients of the 3-point, is the product of $n$ factors of the type $a_{i} b_{k} c_{l}(i \neq k \neq l, i=1,2,3 ; k=1,2,3 ; l=1,2,3)$.

To simplify the proof we write

$$
\begin{array}{ll}
x_{1} b_{2} c_{3}=\alpha_{0}, & a_{1} b_{3} c_{2}=\beta_{0}, \\
a_{2} b_{3} c_{1}=\alpha_{1}, & a_{2} b_{1} c_{3}=\beta_{1}, \\
a_{3} b_{1} c_{2}=\alpha_{2}, & a_{3} b_{2} c_{1}=\beta_{2} .
\end{array}
$$

These $\alpha_{i}$ and $\beta_{i}$ have no relation whatsoever to those used earlier in the article. Clearly as long as every letter and every subscript is present in a term we can continue taking out factors of type $a_{i} b_{k} c_{l}$. But some exponents may be zero. In that case suppose $i_{1}$ say, is not zero. Then the conditions on the exponents give the inequalities:

$$
\begin{array}{rrrrr}
i_{2}<n, & i_{3}<n, & j_{1}<n, & k_{1}<n, \\
j_{2}+k_{2}>0, & j_{3}+k_{3}>0, & j_{2}+j_{3}>0, & k_{2}+k_{3}>0 .
\end{array}
$$

Hence, if $j_{2}=0, k_{2}>0$ and $j_{3}>0$, and therefore $\beta_{0}$ is a factor.

If $k_{3}=0, j_{3}>0, k_{2}>0$ and $\beta_{0}$ is a factor.

If $j_{2}=0$ and $k_{3}=0$ at the same time, $\beta_{0}$ is still a factor.

If $j_{3}=0$ or $k_{2}=0$ or both are zero simultaneously, $\alpha_{0}$ is a factor. Hence, if $i_{1} \neq 0$, either $\alpha_{0}$ or $\beta_{0}$ is a factor. Taking out the factor $\alpha_{0}$ (or $\left.\beta_{0}\right)$, we 
have left a term of same type with $n-1$ replacing $n$. This establishes the lemma and we can write any rational invariant in the form

$$
I=\sum \alpha_{0}^{i} \alpha_{1}^{j} \alpha_{2}^{k} \beta_{0}^{\prime} \beta_{1}^{m} \beta_{2}^{n}
$$

Since $I$ is left unaltered by all permutations of $a, b, c$ and their subscripts, it is left unaltered by all permutations of $\alpha$ and $\beta$ and their subscripts. For if we express the permutations of the Roman letters in cycles, it is easy to express the corresponding permutations of the Greek letters in cyclic form. We have

$$
\begin{aligned}
& (a b c) \text { is equivalent to }\left(\alpha_{0} \alpha_{2} \alpha_{1}\right)\left(\beta_{0} \beta_{1} \beta_{2}\right) \text {, } \\
& (123) \text { " " " }\left(\alpha_{0} \alpha_{1} \alpha_{2}\right)\left(\beta_{0} \beta_{1} \beta_{2}\right) \text {, } \\
& (b c) \text { " " " }\left(\alpha_{0} \beta_{0}\right)\left(\alpha_{1} \beta_{1}\right)\left(\alpha_{2} \beta_{2}\right) \text {, } \\
& (23) \text { " " " }\left(\alpha_{0} \beta_{0}\right)\left(\alpha_{1} \beta_{2}\right)\left(\alpha_{2} \beta_{1}\right) \text {. }
\end{aligned}
$$

This includes all independent types.

Nexi we introduce the notation :

$$
\begin{array}{cc}
\alpha_{0}+\alpha_{1}+\alpha_{2}=r_{1}, & \beta_{0}+\beta_{1}+\beta_{2}=s_{1}, \\
\alpha_{1} \alpha_{2}+\alpha_{2} \alpha_{0}+\alpha_{0} \alpha_{1}=r_{2}, & \beta_{1} \beta_{2}+\beta_{2} \beta_{0}+\beta_{0} \beta_{1}=s_{2}, \\
\alpha_{0} \alpha_{1} \alpha_{2}=p_{3}=\beta_{0} \beta_{1} \beta_{2}, \\
\left(\alpha_{1}-\alpha_{2}\right)\left(\alpha_{2}-\alpha_{0}\right)\left(\alpha_{0}-\alpha_{1}\right)=r, & \left(\beta_{1}-\beta_{2}\right)\left(\beta_{2}-\beta_{0}\right)\left(\beta_{0}-\beta_{1}\right)=\text { s. }
\end{array}
$$

Since any invariant admits of the permutations $\left(\alpha_{c} \alpha_{1} \alpha_{2}\right)$ and $\left(\beta_{0} \beta_{1} \beta_{2}\right)$, it follows that if any invariant $I$ contains the term $\alpha_{0}^{i} \alpha_{1}^{j} \alpha_{2}^{k} \beta_{0}^{l} \beta_{1}^{m} \beta_{2}^{n}$, it contains the 9 terms

$$
\sum^{3} \alpha_{0}^{i} \alpha_{1}^{j} \alpha_{2}^{k} \sum^{3} \beta_{0}^{l} \beta_{1}^{m} \beta_{2}^{n}
$$

$\sum^{3}$ signifying the sum of the three terms obtained by cyclically permuting $i, j$, $k$ and $l, m, n$ respectively. If $i=j=k$ or $l=m=n$, these nine terms reduce to three, or if both sets of equalities exist simultaneously, they reduce to a single term. In any event, we have the product of two alternating functions of $\alpha_{0}$, $\alpha_{1}, \alpha_{2}$ and $\beta_{0}, \beta_{1}, \beta_{2}$ respectively. By the same argument for all terms of $I$, we see that

$$
I=R\left(r_{1}, s_{1}, r_{2}, s_{2}, p_{3}, r, 8\right),
$$

where $R$ is a rational, integral, isobaric function of its arguments. The subscripts indicate the weight, $r$ and $s$ being of weight 3 .

As a next step $r$ and $s$ may be assumed to occur to the first degree only, for $r^{2}$ and $s^{2}$ can both be expressed rationally in terms of the other 5 quantities. In fact*

* Salsion, Higher Algebra, Ex. 2, p. 57. 


$$
\begin{aligned}
& r^{2}=r_{1}^{2} r_{2}^{2}-4 r_{1}^{3} p_{3}+18 r_{1} r_{2} p_{3}-4 r_{2}^{3}-27 p_{3}^{2}, \\
& s^{2}=s_{1}^{2} s_{2}^{2}-4 s_{1}^{3} p_{3}+18 s_{1} s_{2} p_{3}-4 s_{2}^{3}-27 p_{3}^{2} .
\end{aligned}
$$

Also since the permutation $\left(\alpha_{1} \alpha_{2}\right)\left(\beta_{1} \beta_{2}\right)$ changes the sign of $r$ and of $s$, they can occur only in the combination $r s$. Hence we can write

$$
I=R_{1}\left(r_{1}, s_{1}, r_{2}, s_{2}, p_{3}\right)+r s \cdot R_{2}\left(r_{1}, s_{1}, r_{2}, s_{2}, p_{3}\right) \text {. }
$$

Again since $\left(\alpha_{0} \beta_{0}\right)\left(\alpha_{1} \beta_{1}\right)\left(\alpha_{2} \beta_{2}\right)$ interchanges $r_{i}$ and $s_{i}, I$ must be unaltered by interchanging $r_{i}$ and $s_{i}$. That is

$$
R_{1}\left(r_{1}, s_{1}, r_{2}, s_{2}, p_{3}\right)=R_{1}\left(s_{1}, r_{1}, s_{2}, r_{2}, p_{3}\right) \text {. }
$$

Hence if $I$ contains a term $r_{1}^{i} s_{1}^{k} r_{2}^{l} s_{2}^{m} p_{3}^{n}$, it contains the two terms

$$
p_{3}^{n}\left(r_{1} s_{1}\right)^{k}\left(r_{2} s_{2}\right)^{m}\left(r_{1}^{a} r_{2}^{b}+s_{1}^{a} s_{2}^{b}\right)=A_{1},
$$

where for convenience we assume $k \leqq i, m \leqq l, i-k=a, l-m=b$.

Now let

Then

$$
p_{3}^{n}\left(r_{1} s_{1}\right)^{k}\left(r_{2} s_{2}\right)^{m}\left(r_{1}^{a} s_{2}^{b}+s_{1}^{a} r_{2}^{b}\right)=A_{2} .
$$

$$
\begin{aligned}
A_{1}+A_{2} & =p_{3}^{n}\left(r_{1} s_{1}\right)^{k}\left(r_{2} s_{2}\right)^{m}\left(r_{1}^{a}+s_{1}^{n}\right)\left(r_{2}^{b}+s_{2}^{b}\right) \\
& =S_{1}\left(r_{1}+s_{1}, r_{2}+s_{2}, r_{1} s_{1}, r_{2} s_{2}, p_{3}\right), \\
A_{1}-A_{2} & =p_{3}^{n}\left(r_{1} s_{1}\right)^{k}\left(r_{2} s_{2}\right)^{m}\left(r_{1}^{a}-s_{1}^{a}\right)\left(r_{2}^{b}-s_{2}^{b}\right) \\
& =\left(r_{1}-s_{1}\right)\left(r_{2}-s_{2}\right) S_{2}\left(r_{1}+s_{1}, r_{2}+s_{2}, r_{1} s_{1}, r_{2} s_{2}, p_{3}\right),
\end{aligned}
$$

where $S_{1}$ and $S_{2}$ are rational functions. From (6) and (7) we can solve for $A_{1}$ in terms of $r_{1}-s_{1}, r_{2}-s_{2}$ and the arguments of $S_{i}$. Carrying out the same process for all terms of $R_{1}$, we get

$R_{1}=M_{1}\left(r_{1}+s_{1}, r_{2}+s_{2}, r_{1} s_{1}, r_{2} s_{2}, p_{3}\right)+\left(r_{1}-s_{1}\right)\left(r_{2}-s_{2}\right) M_{2}\left(r_{1}+s_{1}, r_{2}+s_{2}, r_{1} s_{1}, r_{2} s_{2}, p_{3}\right)$, where $M_{i}$ is a rational, integral, isobaric function of its arguments. $R_{2}$ can be expressed in the same way. Hence, we have the following theorem.

Any rational simultaneous invariant of $T_{1}$ and $T_{2}$ is a rational, integral isobaric function of $r_{1}+s_{1}, r_{2}+s_{2}, r_{1} s_{1}, r_{2} s_{2}, p_{3},\left(r_{1}-s_{1}\right)\left(r_{2}-s_{2}\right)$, and $r s$.

Since the above seven arguments are themselves invariants, it follows that they form a rationally complete system. They are not independent, however, for we saw that $\overline{r s}^{2}$ could be expressed in terms of the other six. It remains to show that these seven can be expressed in terms of the system proposed in the preceding section. The equalities are easily seen to be

$$
\begin{aligned}
& r_{1}+s_{1}=I_{1} \text {, } \\
& r_{2}+s_{2}=I_{2}, \quad r_{2}-s_{2}=D_{2} \text {, } \\
& p_{3}=I_{3}, \quad r_{1} s_{1}=\frac{1}{4}\left(I_{1}^{2}-D_{1}^{2}\right), \\
& r_{1}-s_{1}=D_{1}, \quad r_{2} s_{2}=\frac{1}{1}\left(I_{2}^{2}-D_{2}^{2}\right) \text {. }
\end{aligned}
$$


The expression for $\overline{r s}$ in terms of the present system is very long. Since $r s$ must be added to our system, for rational completeness we will call it $I_{6}$. In terms of the coefficients of the 3-point

$$
\begin{aligned}
I_{6}=\left(a_{2} b_{3} c_{1}-a_{3} b_{1} c_{2}\right)\left(a_{3} b_{1} c_{2}-a_{1} b_{2} c_{3}\right)\left(a_{1} b_{2} c_{3}-a_{2} b_{3} c_{1}\right) \\
\quad\left(a_{2} b_{1} c_{3}-a_{3} b_{2} c_{1}\right)\left(a_{3} b_{2} c_{1}-a_{1} b_{3} c_{2}\right)\left(a_{1} b_{3} c_{2}-a_{2} b_{1} c_{3}\right) .
\end{aligned}
$$

The completeness of the system has thus been finally established. The principle employed in this paragraph, namely, that if an invariant contains a term $\alpha_{0}^{i} \alpha_{1}^{j} \alpha_{2}^{k} \beta_{0}^{l} \beta_{1}^{m} \beta_{2}^{n}$, it contains all terms obtained by permuting the $i, j, k$ and $l$, $m, n$, is useful in determining all invariant relations, as we need only concern ourselves about a single term of each type.

\section{§3. The dual.forms.}

$\mathrm{W}^{\top}$ e next consider the transformation of the invariants when the rôle of the triangles is interchanged, that is, when the one originally taken as a 3-line is regarded as a 3-point and vice versa. Obviously in the simplified form of the invariants we need only replace each letter by its minor in $D_{1}$. We will designate the transforms by primes. To abbreviate we denote the minor of any letter by the corresponding capital letter.

$$
D_{1}^{\prime}=\left|\begin{array}{lll}
A_{1} & B_{1} & C_{1} \\
A_{2} & B_{2} & C_{2} \\
A_{3} & B_{3} & C_{3}
\end{array}\right|=D_{1}^{2} .
$$

This follows from the theory of 3-rowed determinants.

$$
\begin{aligned}
I_{1}^{\prime}=A_{1} B_{2} C_{3} & +A_{2} B_{3} C_{1}+A_{3} B_{1} C_{2}+A_{1} B_{3} C_{2}+A_{2} B_{1} C_{3}+A_{3} B_{2} C_{1} \\
& =\sum^{3} a_{1}^{2} b_{2}^{2} c_{3}^{2}-\sum^{3} a_{1}^{2} b_{3}^{2} c_{2}^{2}-4\left(\sum^{3} a_{1} a_{2} b_{2} b_{3} c_{3} c_{1}-\sum^{3} a_{1} a_{2} b_{3} b_{1} c_{2} c_{3}\right) .
\end{aligned}
$$

This can be expressed easily, for it is of weight 2 , and the $D$ 's must enter in each term and to an odd degree. This follows from the remark that if the terms of a given type have all the same sign, the $D$ 's must enter to an even degree if at all, but if half the terms are positive and half negative, the $D$ 's must enter in each term and to an odd degree. Hence in the present case we need consider only $I_{1}, D_{1}$, and $D_{2}$. It turns out that

$$
I_{1}^{\prime}=I_{1} D_{1}-6 D_{2} \text {. }
$$

$I_{2}^{\prime}$ and $D_{2}^{\prime}$ stand in a certain formal relation to a square array,

$$
\left|\begin{array}{lll}
A_{2} A_{3} & B_{2} B_{3} & C_{2} C_{3} \\
A_{3} A_{1} & B_{3} B_{1} & C_{3} C_{1} \\
A_{1} A_{2} & B_{1} B_{2} & C_{1} C_{2}
\end{array}\right|
$$


While $D_{2}^{\prime}$ is the determinant of this array, $I_{2}^{\prime}$ is the expanded form with all signs positive. If we designate the sum of the positive terms of $D_{2}^{\prime}$ by $\phi$ and of negative terms by $\theta$,

$$
D_{2}^{\prime}=\phi-\theta, \quad I_{2}^{\prime}=\phi+\theta .
$$

The actual calculation is long and the following table of summations is convenient here and elsewhere :

$$
\begin{aligned}
& \sum^{3} a_{1}^{4} b_{2}^{4} c_{3}^{4}-\sum^{3} a_{1}^{4} b_{3}^{4} c_{2}^{4}=L^{\prime}-L^{\prime \prime} \\
& \sum^{9} a_{1}^{4} b_{2}^{3} b_{3} c_{2} c_{3}^{3}-\sum^{9} a_{1}^{4} b_{2} b_{3}^{3} c_{2}^{3} c_{3}=M^{\prime}-M^{\prime \prime}, \\
& \sum a_{1}^{3} a_{2} b_{1} b_{2}^{2} b_{3} c_{2} c_{3}^{3}-\sum^{9} a_{1}^{3} a_{2} b_{2} b_{3}^{3} c_{1} c_{2}^{2} c_{3}=N^{\prime}-N^{\prime \prime}, \\
& \sum^{18} a_{1}^{3} a_{2} b_{1} b_{2} b_{3}^{2} c_{2}^{2} c_{3}^{2}-\sum^{18} a_{1}^{3} a_{2} b_{2}^{2} b_{3}^{2} c_{1} c_{2} c_{3}^{2}=D^{\prime}-D^{\prime \prime}, \\
& \sum^{3} a_{1}^{2} a_{2}^{2} b_{2}^{2} b_{3}^{2} c_{3}^{2} c_{1}^{2}-\sum^{3} a_{1}^{2} a_{2}^{2} b_{3}^{2} b_{1}^{2} c_{2}^{2} c_{3}^{2}=E^{\prime}-E^{\prime \prime}, \\
& \sum^{3} a_{1}^{2} a_{2} a_{3} b_{1} b_{2}^{2} b_{3} c_{1} c_{2} c_{3}^{2}-\sum^{3} a_{1}^{2} a_{2} a_{3} b_{1} b_{2} b_{3}^{2} c_{1} c_{2}^{2} c_{3}=F^{\prime \prime}-F^{\prime \prime} \text {, } \\
& \sum^{6} a_{1}^{3} a_{2} b_{2}^{3} b_{3} c_{3}^{3} c_{1}-\sum^{6} a_{1}^{3} a_{2} b_{3}^{3} b_{1} c_{2}^{3} c_{3}=G^{\prime \prime}-G^{\prime \prime}, \\
& \sum^{9} a_{1}^{2} a_{2}^{2} b_{1} b_{2} b_{3}^{2} c_{1} c_{2} c_{3}^{2}=H, \\
& \sum^{9} a_{1}^{4} b_{2}^{2} b_{3}^{2} c_{2}^{2} c_{3}^{2}=K \text {. }
\end{aligned}
$$

Further let $L^{\prime}+L^{\prime \prime}=L$; etc. In this notation, there is no difficulty beyond long reckoning. Less direct methods offered no advantage. We have then

Hence

$$
\begin{aligned}
& \phi=E^{\prime}+3 E^{\prime \prime}-2 D^{\prime}+2 N^{\prime}+N^{\prime \prime}-5 F^{\prime \prime}+6 F^{\prime}-G^{\prime}, \\
& \theta=3 E^{\prime}+E^{\prime \prime}-2 D^{\prime \prime}+N^{\prime}+2 N^{\prime \prime}+6 F^{\prime \prime}-5 F^{\prime \prime}-G^{\prime \prime} .
\end{aligned}
$$

$$
\begin{aligned}
& I_{2}^{\prime}=4 E-2 D+3 N+F-G, \\
& D_{2}^{\prime}=-2\left(E^{\prime}-E^{\prime \prime}\right)-2\left(D^{\prime}-D^{\prime \prime}\right)+\left(N^{\prime}-N^{\prime \prime}\right)-11\left(F^{\prime}-F^{\prime \prime}\right)-\left(G^{\prime}-G^{\prime \prime}\right) .
\end{aligned}
$$

In $I_{2}^{\prime}$ the $D$ 's must enter to an even degree, if at all. It is readily shown that

and from these

$$
\begin{aligned}
D_{1}^{2} I_{2} & =N-2 D+2 E-F+G+4 H, \\
I_{1} D_{1} D_{2} & =-N+2 E+5 F+G-4 H, \\
D_{2}^{2} & =E+2 F-2 H,
\end{aligned}
$$

$$
I_{2}^{\prime}=D_{1}^{2} I_{2}-2 I_{1} D_{1} D_{2}+6 D_{2}^{2} \text {. }
$$


$D_{2}^{\prime}$ must contain the $D$ 's to odd degree in every term. It turns out at once that

$$
\begin{aligned}
D_{2}^{\prime} & =-D_{1}^{2} D_{2}, \\
I_{3}^{\prime} & =A_{1} A_{2} A_{3} B_{1} B_{2} B_{3} C_{1} C_{2} C_{3} .
\end{aligned}
$$

The actual expansion and reduction of this form is too long for reproduction here, but we get as a final form

$$
I_{3}^{\prime}=D_{1}^{3} I_{3}-D_{2}^{3}+\frac{1}{2} D_{1} D_{2}\left(I_{1} D_{2}-I_{2} D_{1}\right)
$$

Summarizing the results of this section into a compact table, we have:

$$
\begin{aligned}
I_{1}^{\prime} & =I_{1} D_{1}-6 D_{2}, \\
D_{1}^{\prime} & =D_{1}^{2}, \\
I_{2}^{\prime} & =D_{1}^{2} I_{2}-2 I_{1} D_{1} D_{2}+6 D_{2}^{2}, \\
D_{2}^{\prime} & =-D_{1}^{2} D_{2}, \\
I_{3}^{\prime} & =D_{1}^{3} I_{3}-D_{2}^{3}+\frac{1}{2} D_{1} D_{2}\left(I_{1} D_{2}-I_{2} D_{1}\right)
\end{aligned}
$$

It is a simple matter to write down the corresponding table when the 3-line is taken in general form.*

These formulæ are very useful, for if we know that the vanishing of an invariant $I$ indicates a certain projective relation between the 3 -line and the 3-point, the vanishing of $I^{\prime}$, obtained from $I$ by these formulæ, will indicate the same relation between the joins of the 3-point and the meets of the 3-line.

This dual transformation, being obviously of period 2, affords an easy check on the algebra involved in calculating the dual forms. Carrying out the substitutions of $(I)$ a second time we get

$$
\begin{aligned}
& I_{1}^{\prime}=D_{1}^{2}\left(I_{1} D_{1}-6 D_{2}\right)+6 D_{1}^{2} D_{2}=D_{1}^{3} I_{1}, \\
& D_{1}^{\prime \prime}=D_{1}^{4}, \quad D_{2}^{\prime \prime}=D_{1}^{6} D_{2}, \\
& I_{2}^{\prime \prime}=D_{1}^{6} I_{2} ; \quad I_{3}^{\prime \prime}=D_{1}^{9} I_{3} .
\end{aligned}
$$

This is an excellent verification of the dual formulæ.

§4. Some invariant relations of the three-point and the three-line.

We shall calculate directly a few of the simpler invariant relations.

(1) The three points are collinear if

$$
D_{1}=0 \text {. }
$$

* For these forms, see the Johns Hopkins University Circular, July, 1908, p. 125. 
(2) The three lines are concurrent if

$$
\Delta_{1}=0 \text {. }
$$

The above two theorems require no formal proof.

(3) The 3-point is apolar to the 3-line if

$$
I_{1}=0 \text {. }
$$

For $x_{1} x_{2} x_{3}=0$ is the 3-line and $(a \xi)(b \xi)(c \xi)=0$ the 3-point. Regarding the $x$ 's as differential operators, and operating on the equation of the 3-point, we get merely the coefficient of $\xi_{1} \xi_{2} \xi_{3}$ in the equation, which must be zero for apolarity. This is,

$$
a_{1} b_{2} c_{3}+a_{2} b_{3} c_{1}+a_{3} b_{1} c_{2}+a_{1} b_{3} c_{2}+a_{2} b_{1} c_{3}+c_{3} b_{2} c_{1}=0 .
$$

But that is precisely

$$
I_{1}=0 \text {. }
$$

(4) The three points of the 3-point and the meets of the 3-line are on a conic ị

$$
D_{2}=0 \text {. }
$$

For the general conic on the meets of the 3-line, i. e., on the vertices of the reference triangle, is

$$
\alpha_{1} x_{2} x_{3}+\alpha_{2} x_{3} x_{1}+\alpha_{3} x_{1} x_{2}=0 .
$$

If the points $(a \xi)=0,(b \xi)=0$ and $(c \xi)=0$ are on this conic;

$$
\begin{aligned}
& \alpha_{1} a_{2} a_{3}+\alpha_{2} a_{1} a_{3}+\alpha_{3} a_{1} a_{2}=0, \\
& \alpha_{1} b_{2} b_{3}+\alpha_{2} b_{1} b_{3}+\alpha_{3} b_{1} b_{2}=0, \\
& \alpha_{1} c_{2} c_{3}+\alpha_{2} c_{1} c_{3}+\alpha_{3} c_{1} c_{2}=0 .
\end{aligned}
$$

For these 3 equations to be consistent, their determinant must vanish. That is

$$
\left|\begin{array}{ccc}
a_{2} a_{3} & a_{3} a_{1} & a_{1} a_{2} \\
b_{2} b_{3} & b_{3} b_{1} & b_{1} b_{2} \\
c_{2} c_{3} & c_{3} c_{1} & c_{1} c_{2}
\end{array}\right|=0,
$$

which is $D_{2}=0$. For the general case, a study of the degree in the Greek letters shows that

is the general condition.

$$
\Delta_{1}^{2} D_{2}=0
$$

The dual of $D_{2}, D_{1}^{2} D_{2}=0$, is therefore the condition that the 6 lines be on a conic.

(5) We will next take the problem of $\mathrm{P}_{\mathrm{ASCH}}$ * triangles and prove: If the

* Mathematisohe Annalen, Vol. 23 (1881), p. 426 ff. See also Hus, these Transactions, Vol. 5 (1904), p. 49. Pasch shows that if there is one such triangle in a collineation, there is a quadruple infinity. 
two triangles are such that the 3-point is a $\mathrm{P}_{\mathrm{ASCH}}$ triangle in a normal collineation having the 3-line for a fixed triangle,

$$
D_{1}\left(I_{1} D_{1}-3 D_{2}\right)=0 \text {. }
$$

To shcw this, we take again the 3-line as reference triangle. . The normal collineation can be written

$$
x_{i}=k a_{i}
$$

and we want the transform of $a$ to be on the line $b c$. Similarly $b$ is to go into a point on $a c$, and $c$ into a point on $a b$. This gives us three equations like

We can write them

$$
\left|\begin{array}{rrrr}
k_{1} a_{1} & k & a_{2} & k_{3} a_{3} \\
b_{1} & b_{2} & b_{3} \\
c_{1} & c_{2} & c_{3}
\end{array}\right|=0 .
$$

$$
\begin{aligned}
& k_{1} a_{1} A_{1}+k_{2} a_{2} A_{2}+k_{3} a_{3} A_{3}=0, \\
& k_{1} b_{1} B_{1}+k_{2} b_{2} B_{2}+k_{3} b_{3} B_{3}=0, \\
& k_{1} c_{1} C_{1}+k_{2} c_{2} C_{2}+k_{3} c_{3} C_{3}=0,
\end{aligned}
$$

where the $A_{i}, B_{i}, C_{i}$, are minors as before.

For these 3 equations to be consistent, we must have

Expanding this we get

$$
\left|\begin{array}{lll}
a_{1} A_{1} & a_{2} A_{2} & a_{3} A_{3} \\
b_{1} B_{1} & b_{2} B_{2} & b_{3} B_{3} \\
c_{1} C_{1} & c_{2} C_{2} & c_{3} C_{3}
\end{array}\right|=0
$$

$$
\sum^{6} a_{1}^{3} b_{2}^{3} c_{3}^{3}-\sum^{18} a_{1}^{3} b_{2}^{2} b_{3} c_{2} c_{3}^{2}+\sum^{18} a_{1}^{2} a_{3} b_{2}^{2} b_{3} c_{1} c_{2} c_{3}-6 a_{1} a_{2} a_{3} b_{1} b_{2} b_{3} c_{1} c_{2} c_{3}=0 .
$$

This expresses itself readily in the form

$$
I D_{1}^{2}-3 D_{1} D_{2}=0, \text { or } D_{1}\left(I_{1} D_{1}-3 D_{2}\right)=0,
$$

as we were to prove.

(6) If the two triangles are in perspective position,

$$
I_{6}=0 \text {. }
$$

It will be found that the 6 factors of $I_{6}$ correspond to the 6 possible orderings of the vertices of the two triangles. For example, by way of proof, suppose we ask that the joins of $1,0,0 ; 0,1,0 ; 0,0,1$ to $a ; b ; c$, respectively meet in a point. The 3 joins are

$$
\begin{aligned}
& a_{2} x_{3}-a_{3} x_{2}=0, \\
& b_{3} x_{1}-b_{1} x_{3}=0, \\
& c_{1} x_{2}-c_{2} x_{1}=0 .
\end{aligned}
$$


Hence the condition that they meet in a point is

$$
\left|\begin{array}{ccc}
0 & -a_{3} & a_{2} \\
b_{3} & 0 & -b_{1} \\
-c_{2} & c_{1} & 0
\end{array}\right|=0,
$$

or $a_{2} b_{3} c_{1}-a_{3} b_{1} c_{2}=0$. In the same way each ordering requires a disţinct factor of $I_{6}$ to vanish. As a corollary we can also state: If there exists a conic with respect to which the 3-line and 3-point are mutually polar, then must

$$
I_{6}=0 \text {. }
$$

All the problems discussed by Dr. Hun might be treated independently as those of this paragraph have been. But every fundamental system of invariants must be expressible in terms of the present system, since it has been proven complete. Hence by constructing a table of equivalents we can utilize directly Dr. Hus's results.

\section{\$5. Hun's invariants in terms of the present system.}

Hus's three fundamental invariants are defined for the case of a general 3-line and 3-point as follows:

$$
\begin{aligned}
& I_{1}=-\sum B_{11}, \\
& I_{2}=\sum\left(\begin{array}{lll}
B_{22} & \left.B_{33}-B_{23} B_{32}\right), \\
B_{11} & B_{12} & B_{13} \\
I_{3}=-B_{21} & B_{22} & B_{23} \\
B_{31} & B_{32} & B_{33}
\end{array}\right.
\end{aligned}
$$

where

$$
\begin{aligned}
& B_{i i}=\alpha_{i}(\beta \gamma)_{i}+\beta_{i}(\gamma \alpha)_{i}+\gamma_{i}(\alpha \beta)_{i}, \\
& B_{i k}=\alpha_{i}(\beta \gamma)_{k}+\beta_{i}(\gamma \alpha)_{k}+\gamma_{i}(\alpha \beta)_{k},
\end{aligned}
$$

and in turn the symbols $(\beta \gamma)_{i},(\gamma x)_{k}$, etc., are defined as follows:

where finally

$$
(\beta \gamma)_{i}=a_{i}(b c / \beta \gamma)+b_{i}(c a / \beta \gamma)+c_{i}(a b / \beta \gamma) ;
$$

$$
\begin{aligned}
& (b c / \beta \gamma)=(b \beta)(c \gamma)+(b \gamma)(c \beta), \\
& (c a / \beta \gamma)=(c \beta)(c \gamma)+(c \gamma)(a \beta), \text { etc., }
\end{aligned}
$$

the parentheses now indicating row products.

If now the 3-line is taken as the reference triangle, we need retain only terms containing the product $\alpha_{1} \beta_{2} \gamma_{3}$ and replace it by unity. This gives

$$
B_{11}=B_{22}=B_{33}=\sum^{6}{ }^{6}{ }_{i} b_{k} c_{l} \text {, }
$$


In general

$$
\begin{aligned}
& B_{21}=2\left(a_{1} b_{1} c_{3}+a_{1} b_{3} c_{1}+a_{3} b_{1} c_{1}\right), \\
& B_{12}=2\left(a_{2} b_{2} c_{3}+a_{2} b_{3} c_{2}+a_{3} b_{2} c_{2}\right) .
\end{aligned}
$$

$$
B_{i j}=2\left(a_{j} b_{j} c_{k}+a_{j} b_{k} c_{j}+a_{k} b_{j} c_{j}\right) \text {. }
$$

Then denoting Hus's invariants by bars to avoid confusion, we have

$$
\begin{aligned}
\bar{I}_{1}=- & \sum^{3} B_{i i}=-3 \sum^{6} a_{i} b_{k} c_{2}=-3 I_{1}, \\
\bar{I}_{2}=\sum^{3} & \left(B_{22} B_{33}-B_{23} B_{32}\right)=3\left(\sum^{6} a_{i} b_{k} c_{2}\right)^{2} \\
& \quad-4\left[\left(a_{3} b_{3} c_{1}+a_{3} b_{1} c_{3}+a_{1} b_{3} c_{3}\right)\left(a_{2} b_{2} c_{1}+a_{2} b_{1} c_{2}+a_{1} b_{2} c_{2}\right)\right. \\
& +\left(a_{3} b_{3} c_{2}+a_{3} b_{2} c_{3}+a_{2} b_{3} c_{3}\right)\left(a_{1} b_{1} c_{2}+a_{1} b_{2} c_{1}+a_{2} b_{1} c_{1}\right) \\
& \left.\quad+\left(a_{2} b_{2} c_{3}+a_{2} b_{3} c_{2}+a_{3} b_{2} c_{2}\right)\left(a_{1} b_{1} c_{3}+a_{1} b_{3} c_{1}+a_{3} b_{1} c_{1}\right)\right] \\
= & 3 I_{1}^{2}-4\left(\sum^{0} a_{1}^{2} b_{2} b_{3} c_{2} c_{3}+3 \sum^{6} a_{2} a_{3} b_{3} b_{1} c_{1} c_{2}\right) \\
= & 3 I_{1}^{2}-\left(I_{1}^{2}-D_{1}^{2}+12 I_{2}\right)=2 I_{1}^{2}+D_{1}^{2}-12 I_{2} .
\end{aligned}
$$

The transformation of $-I_{3}=-\bar{\Delta} \bar{D} \bar{N}$ is more difficult. Obviously $\bar{\Delta}$ and $\bar{D}$ are $\Delta_{1}$ and $D_{1}$ respectively. We have directly from above equalities (1),

$$
\begin{array}{ccc}
I_{1} & 2\left(a_{2} b_{2} c_{3}+a_{2} b_{3} c_{2}+a_{3} b_{2} c_{2}\right) & 2\left(a_{3} b_{3} c_{2}+a_{3} b_{2} c_{3}+a_{2} b_{3} c_{3}\right) \\
-I_{3}=2\left(a_{1} b_{1} c_{3}+a_{1} b_{3} c_{1}+a_{3} b_{1} c_{1}\right) & I_{1} & 2\left(a_{3} b_{3} c_{1}+a_{3} b_{1} c_{3}+a_{1} b_{3} c_{3}\right) . \\
2\left(a_{1} b_{1} c_{2}+a_{1} b_{2} c_{1}+a_{2} b_{1} c_{1}\right) & 2\left(a_{2} b_{2} c_{1}+a_{2} b_{1} c_{2}+a_{1} b_{2} c_{2}\right) & I_{1}
\end{array}
$$

Expanding this and using the equality found in evaluating $\bar{I}_{2}$, we have

$$
\begin{aligned}
-\bar{I}_{3}=I_{1}^{3} & +16 \sum^{18} a_{1} a_{2} a_{3} b_{2} b_{3}^{2} c_{1}^{2} c_{2}+8 \sum^{12} a_{2} a_{3}^{3} b_{3} b_{1}^{2} c_{1} c_{2}^{3} \\
& +48 a_{1} a_{2} a_{3} b_{1} b_{2} b_{3} c_{1} c_{2} c_{3}-I_{1}\left(I_{1}^{2}-D_{1}^{2}+12 I_{2}\right) \\
= & I_{1}^{3}+12 I_{1} I_{2}-4 D_{1} D_{2}-I_{1}^{3}+I_{1} D_{1}^{2}-12 I_{1} I_{3}=D_{1}\left(I_{1} D_{1}-4 D_{2}\right)
\end{aligned}
$$

Hence

$$
\bar{N}=4 D_{2}-I_{1} D_{1}
$$

Therefore we have as a complete table

$$
\begin{array}{ll}
D=D_{1}, & \bar{I}_{2}=2 I_{1}^{2}+D_{1}^{2} \Delta_{1}^{2}-12 I_{2}, \\
\bar{\Delta}=\Delta_{1}, & \bar{V}=-I_{1} D_{1} \Delta_{1}+4 D_{2}, \\
& \bar{I}_{1}=-3 I_{1},
\end{array}
$$

where the $\Delta_{1}$ 's are inserted 80 as to be correct for the general 3-line. 
Using these formulas we shall write down the equivalents of Dr. Irv's results as tabulated on pages 50 and 51 of these Transactions, vol. 5 (1904).

The 3-point degenerates, if

$$
D_{1}=0 \text {. }
$$

The 3-point is apolar to the 3-line, it

$$
I_{1}=0 \text {. }
$$

The meets of the 3-line are apolar to the joins of the 3-point, if

$$
I_{1} D_{1} \Delta_{1}-6 D_{2}=0 \text {. }
$$

A conic circumscribed to the 3-point, and apolar to the 3-line exists, if

$$
I_{1} \Delta_{1} D_{1}-2 D_{2}=0 \text {. }
$$

A point conic apolar to both the 3 point and the 3-line exists, if

$$
\eta_{1}^{2} \eta_{2}=0 \text {. }
$$

A conic circumscribed about both the 3-point and the 3-line exists, if

$$
\Delta_{1}^{2} D_{2}=0 \text {. }
$$

There exists a point, whose polar conic as to the 3 -line is apolar to the 3-point, if

$$
D_{1} \Delta_{1}\left(I_{1} D_{1} \Delta_{1}-4 D_{2}\right)=0 .
$$

The three polar lines as to the 3 . line, of the points of the 3-point, taken two at a time, meet in a point, if

$$
\Delta_{1}\left(I_{1} D_{1} \Delta_{1}-4 D_{2}\right)=0 \text {. }
$$

There exists a collineation having the 3-point as a fixed triangle, which sends each meet of the 3-line into a point on the opposite line, if

$$
\Delta_{1}\left(I_{1} D_{1} \Delta_{1}-3 D_{2}\right)=0 \text {. }
$$

There exists a collineation having the meets of the 3-line as fixed points, which sends each point of the 3-point into a
'The 3-line degenerates, if

$$
\Delta_{1}=0 \text {. }
$$

The 3-line is apolar to the 3-point, if

$$
I_{1}=0 \text {. }
$$

The joins of the 3-point are apolar to the meets of the 3-line, if

$$
I_{1} D_{1} \Delta_{1}-6 D_{2}=0 \text {. }
$$

I conic inscribed in the 3-line and apolar to the 3-point exists, if

$$
I_{1} I_{1} \Delta_{1}-2 I_{2}=0 \text {. }
$$

A line ronic apolar to both the 3 . line and the 3-point exists, if

$$
\Delta_{1}^{2} D_{2}=0 \text {. }
$$

A conic inseribed to both the 3-point and the 3-line exists, if

$$
D_{1}^{:} D_{2}=0 \text {. }
$$

There exists a line, whose polar conic as to the 3-point is apolar to the 3 -line, if

$$
D_{1} \Delta_{1}\left(I_{1} D_{1} \Delta_{1}-4 D_{2}\right)=0 \text {. }
$$

The three polar points as to the 3 point, of the lines of the 3 -line, taken two at a time, lie on a line, if

$$
D_{1}\left(I_{1} D_{1} \Delta_{1}-4 D_{2}\right)=0 \text {. }
$$

There exists a collineation having the 3-line as a fixed triangle, which sends each join of the 3-point into a line through the opposite point, if

$$
D_{1}\left(I_{1} D_{1} \Delta_{1}-3 D_{2}\right)=0 \text {. }
$$

There exists a collineation having the joins of the 3-point as fixed lines, which sends each line of the 3 -line into a line 
point on the join of the other two, if

$$
I_{1} D_{1} \Delta_{1}-3 D_{2}=0 \text {. }
$$

There exists a point, such that its polar conic as to the joins of the 3point is apolar to the meets of the 3 line, if

$$
D_{1}^{2} \Delta_{1}^{2}\left(I_{1} D_{1} \Delta_{1}-2 D_{2}\right)=0
$$

through the meet of the other two, if

$$
I_{1} D_{1} \Delta_{1}-3 D_{2}=0 \text {. }
$$

There exists a line such that its polar conic as to the meets of the 3 . line is apolar to the joins of the 3 . point, if

$$
D_{1}^{2} \Delta_{1}^{2}\left(I_{1} D_{1} \Delta_{1}-2 D_{2}\right)=0 .
$$

To this we will add one more, adapted from the same article.

There exists a line conic, touching the joins of the 3-point, and apolar to the 3-line, if

$$
D_{1}^{2}\left(I_{1} D_{1} \Delta_{1}-4 D_{2}\right)=0 \text {. }
$$

It will be seen that the essential factor of all these invariants is a member of the pencil,

$$
I_{1} D_{1} \Delta_{1}+\lambda \dot{D}_{2}=0 \text {. }
$$

It may be noted that if two points of the 3-point are taken as the circular points at infinity, and the third point taken as variable, this pencil is the pencil of circles determined by the Fenerbach circle and the circumcircle.

Another pair of dual theorems which can be readily proven are these:

If one of the 3 points lies on either of the lines of the 3-line,

$$
I_{3}=0 \text {. }
$$

If a meet of the 3-line lies on a join of the 3-point,

$$
2 D_{1}^{3}-2 D_{2}^{3}+D_{1} D_{2}\left(I_{1} D_{2}-I_{2} D_{1}\right)=0 \text {. }
$$

The powers of $\Delta_{1}$ are not inserted in the latter form, and will not be in the future unless for some special reason.

\section{\$6. A conic inscribed in one triangle and circumscribed about the other.}

The present investigation has grown by extension from the problem: To find the invariant relation on two triangles so that a conic may be drawn touching the sides of one triangle and on the vertices of the other. This problem together with its applications is especially interesting.

Let the three lines be the reference triangle, and take the polar conics of the three points $a, b, c$ as to it. The three polar conics are:

$$
\begin{aligned}
& a_{1} x_{2} x_{3}+a_{2} x_{3} x_{1}+a_{3} x_{1} x_{2}=0, \\
& b_{1} x_{2} x_{3}+b_{2} x_{3} x_{1}+b_{3} x_{1} x_{2}=0, \\
& c_{1} x_{2} x_{3}+c_{2} x_{3} x_{1}+c_{3} x_{1} x_{2}=0 .
\end{aligned}
$$


Let $\eta$ be any line of (1), the polar conic of $a$, and the poloconic of $\eta$ will pass throngh $a .^{*}$ Ilence if the three polar conies have $\eta$ as a common line, then the poloconic of $\eta$ as to the 3-line will be on all three points $a, b, c$. But it will also touch the three reference lines $\alpha, \beta, \gamma$, for the Ilessian of a triangle is the triangle itself, and the poloconic of any cubic tonches the Hessian in three points $\dagger$ : in the present case it tonches each of the three lines making up the Hessian, for they enter symmetrically.

Conseguently the problem is now reduced to finding the condition that the three polar conics have a common line. Salyox $\ddagger$ gives the condition that three point conics have a common point as $T^{2}=64.1 Y$. For three conics to have a common line we need only form their line equations and impose the analogous condition. The line equations of $(1),(2),(3)$ are

$$
\begin{aligned}
& a_{1}^{2} \xi_{1}^{2}+a_{2}^{2} \xi_{2}^{2}+a_{3}^{2} \xi_{3}^{2}-2 a_{2} a_{3} \xi_{2} \xi_{3}-2 a_{3} a_{1} \xi_{3} \xi_{1}-2 a_{1} a_{2} \xi_{1} \xi_{2}=0=C . \\
& l_{1}^{2} \xi_{1}^{2}+b_{2}^{2} \xi_{2}^{2}+b_{3}^{2} \xi_{3}^{2}-2 b_{2} b_{3} \xi_{2} \xi_{3}-2 b_{3} b_{1} \xi_{3} \xi_{1}-2 b_{1} b_{2} \xi_{1} \xi_{2}=0=V, \\
& c_{1}^{2} \xi_{1}^{2}+c_{2}^{2} \xi_{2}^{2}+c_{3}^{2} \xi_{3}^{2}-2 c_{2} c_{3} \xi_{2} \xi_{3}-2 c_{3} c_{1} \xi_{3} \xi_{1}-2 c_{1} c_{2} \xi_{1} \xi_{2}=0=W .
\end{aligned}
$$

Now $M=0$ is the condition that in a net of line conics $l U+m V+n W$ there shall be a double point. In that case, the reciprocal of that particular conic of the net vanishes identically. Following SaLxox's notation, $\S$ we find the equation to be

$$
l^{2} \sum+m^{2} \Sigma^{\prime}+n^{2} \Sigma^{\prime \prime}+m n \phi_{23}+n l \phi_{31}+l m \phi_{12}=0 .
$$

The $\sum$ 's are the reciprocals of $C, V, W$ respectively and the $\phi_{i k}$ are the Clebschians of the same taken two at a time :

$$
\Sigma=4 a_{1} a_{2} u_{3}\left(a_{1} \cdot x_{2} x_{3}+a_{2} \cdot r_{3} x_{1}+a_{3} x_{1} x_{2}\right)=0 .
$$

$\Sigma^{\prime}$ and $\Sigma^{\prime \prime}$ are the same in $b$ and $c$ respectively. By the Clebschian of two line conics $(a \xi)^{2}$ and $(b \xi)^{2}$, we mean the contravariant $\mid a b{ }^{2}{ }^{2}$, where $|a b x|$ is the determinant. $\|$ In the present case

$$
\begin{aligned}
\phi_{23}= & \left(a_{2} b_{3}-a_{3} b_{2}\right)^{2} x_{1}^{2}+\left(a_{3} b_{1}-a_{1} b_{3}\right)^{2} x_{2}^{2}+\left(a_{1} b_{2}-a_{2} b_{1}\right)^{2} x_{3}^{2} \\
& +\left(a_{3} b_{1}-a_{1} b_{3}\right)\left(a_{1} b_{2}-a_{2} b_{1}\right) x_{2} x_{3}+\left(a_{1} b_{2}-a_{2} b_{1}\right)\left(a_{2} b_{3}-a_{3} b_{2}\right) x_{1} x_{3} \\
& +\left(a_{2} b_{3}-a_{3} b_{2}\right)\left(a_{3} b_{1}-a_{1} b_{3}\right) x_{1} x_{2},
\end{aligned}
$$

* Salyox-Fienleb, - Anclytische Gometic der hïleirn ebenen Kurten, p. 292. The English edition of this book is out of print. SALMos calls the poloconic simply the polar conic of the line. Clebsch-Lrspexasv, - Vorlesungen über Geometrie, Vol. I, p. 543. Kons, Encyklopülie dei mathematischen Wissenschuften, Vol. III, part 4, p. 471, gives an account of the poloconic with references.

† DURÈGe: Ẻbene C'urren diitter Orcinung, p. 278.

$\$$ Conic Sections, p. 365-6.

Sonic Sections, p. 366.

il Ibid., p. 344. Chebsch's form is non-symbolic and $o$ equals $o_{12}$ abore. See also ClebschLisnexasi, Vol. I, p. 277. If $a_{i} \equiv b_{i}$, the Clebschian is the line equation.

Traus. Am. Math. Soc. 28 
and $\phi_{31}$ and $\phi_{12}$ are similar forms in $b, c$ and $c, a$ respectively. The above forms, $\sum$ and $\phi_{i k}$, are most easily verified by expanding the symbolic forms and substituting the real coefficients.

Salmon gives $M$ in the form of a 6-rowed determinant. From equations (8) we see that the block

$$
\left|\begin{array}{lll}
A & B & C \\
A^{\prime} & B^{\prime} & C^{\prime} \\
A^{\prime \prime} & B^{\prime \prime} & C^{\prime \prime}
\end{array}\right|
$$

is composed entirely of zeros. Hence $M$ reduces at once to the product of two 3-rowed determinants

$$
\left|\begin{array}{lll}
2 a_{1}^{2} a_{2} a_{3} & 2 a_{1} a_{2}^{2} a_{3} & 2 a_{1} a_{2} a_{3}^{2} \\
2 b_{1}^{2} b_{2} b_{3} & 2 b_{1} b_{2}^{2} b_{3} & 2 b_{1} b_{2} b_{3}^{2} \\
2 c_{1}^{2} c_{2} c_{3} & 2 c_{1} c_{2}^{2} c_{3} & 2 c_{1} c_{2} c_{3}^{2}
\end{array}\right| \times \mid \begin{array}{lll}
\left(a_{2} b_{3}-a_{3} b_{2}\right)^{2} & \left(a_{3} b_{1}-a_{1} b_{3}\right)^{2} & \left(a_{1} b_{2}-a_{2} b_{1}\right)^{2} \\
\left(b_{2} c_{3}-b_{3} c_{2}\right)^{2} & \left(b_{3} c_{1}-b_{1} c_{3}\right)^{2} & \left(b_{1} c_{2}-b_{2} c_{1}\right)^{2} \\
\left(c_{2} a_{3}-c_{3} a_{2}\right)^{2} & \left(c_{3} a_{1}-c_{1} a_{3}\right)^{2} & \left(c_{1} a_{2}-c_{2} a_{1}\right)^{2}
\end{array}
$$

The first determinant is clearly $8 I_{3} D_{1}$. The second is seen to be the dual of

$$
\left|\begin{array}{lll}
c_{1}^{2} & c_{2}^{2} & c_{3}^{2} \\
a_{1}^{2} & a_{2}^{2} & a_{3}^{2} \\
b_{1}^{2} & b_{2}^{2} & b_{3}^{2}
\end{array}\right|
$$

Now (11) in terms of our fundamental invariants is

$$
I_{1} D_{1}-2 D_{2} \text {. }
$$

The dual of this, by the formulæ of $(\mathrm{I}), \S 3$, is

Therefore

$$
D_{1}^{2}\left(I_{1} D_{1}-4 D_{2}\right) \text {. }
$$

$$
M=8 I_{3} D_{1}^{3}\left(I_{1} D_{1}-4 D_{2}\right) \text {. }
$$

To express $T$ similarly we can start with either of SALMON's forms as given on pages 365 and 367 of his Conic Sections. We shall develop the second form

$$
T=\theta_{123}^{2}-4\left(\theta_{122} \theta_{133}+\theta_{211} \theta_{233}+\theta_{311} \theta_{322}\right)+12 \Theta .
$$

Next recall the meaning of the $\theta$ 's. If we write the discriminant of

$$
l(a \xi)^{2}+m(b \xi)^{2}+n(c \xi)^{2}
$$

we can define them as follows: $\theta_{123}$ is the coefficient of $l m n$ in the discriminant, $\theta_{122}$ is the coefficient of $l m^{2}$, and so on. For the net $l U+m V+n W$, this gives

$$
\theta_{123}=\sum^{6} a_{1}^{2} b_{2}^{2} c_{3}^{2}-2 \sum^{6} a_{1} a_{2} b_{2} b_{3} c_{3} c_{1}-2 \sum^{9} a_{1}^{2} b_{2} b_{3} c_{2} c_{3}=D_{1}^{2}-4 I_{2},
$$




$$
\begin{aligned}
& \theta_{122}=-4\left(a_{1} a_{2} b_{1} b_{2} b_{3}^{2}+a_{2} a_{3} b_{2} b_{3} b_{1}^{2}+a_{3} a_{1} b_{1} b_{2}^{2} b_{3}\right), \\
& \theta_{133}=-4\left(a_{1} a_{2} c_{1} c_{2} c_{3}^{2}+a_{2} a_{3} c_{1}^{2} c_{2} c_{3}+a_{3} a_{1} c_{1} c_{2}^{2} c_{3}\right),
\end{aligned}
$$

and similarly for $\theta_{211}, \theta_{233}, \theta_{311}, \theta_{322}$. Combining these we have $\theta_{192} \theta_{133}=16 .\left(a_{1}^{2} a_{2}^{2} b_{1} b_{2} b_{3}^{2} c_{1} c_{2} c_{3}^{2}+a_{2}^{2} a_{3}^{2} b_{1}^{2} b_{2} b_{3} c_{1}^{2} c_{2} c_{3}\right.$

$$
\left.+a_{3}^{2} a_{1}^{2} b_{1} b_{2}^{2} b_{3} c_{1} c_{2}^{2} c_{3}+\sum^{6} a_{1}^{2} a_{2} a_{3} b_{1} b_{2}^{2} b_{3} c_{1} c_{2} c_{3}^{2}\right),
$$

$\theta_{211} \theta_{233}=16\left(a_{1} a_{2} a_{3}^{2} b_{1}^{2} b_{2}^{2} c_{1} c_{2} c_{3}^{2}+a_{1}^{2} a_{2} a_{3} b_{2}^{2} b_{3}^{2} c_{1}^{2} c_{2} c_{3}\right.$

$$
\left.+a_{1} a_{2}^{2} a_{3} b_{3}^{2} b_{1}^{2} c_{1} c_{2}^{2} c_{3}+\sum^{6} a_{1}^{2} a_{2} a_{3} b_{1} b_{2}^{2} b_{3} c_{1} c_{2} c_{3}^{2}\right),
$$

$\theta_{311} \theta_{322}=16\left(a_{1} a_{2} a_{3}^{2} b_{1} b_{2} b_{3}^{2} c_{1}^{2} c_{2}^{2}+a_{1}^{2} a_{2} a_{3} b_{1}^{2} b_{2} b_{3} c_{2}^{2} c_{3}^{2}\right.$

Hence

$$
\left.+a_{1} a_{2}^{2} a_{3} b_{1} b_{2}^{2} b_{3} c_{3}^{2} c_{2}^{2}+\sum^{6} a_{1}^{2} a_{2} a_{3} b_{1} b_{2}^{2} b_{3} c_{1} c_{2} c_{3}^{2}\right) \text {. }
$$

$$
\begin{aligned}
\theta_{122} \theta_{133}+\theta_{211} \theta_{233}+\theta_{311} \theta_{322}=16\left(\sum^{9} a_{1}^{2} a_{2}^{2} b_{1} b_{2} b_{3}^{2} c_{1} c_{2} c_{3}^{2}+3\right. & \left.\sum^{6} a_{1}^{2} a_{2} a_{3} b_{1} b_{2}^{2} b_{3} c_{1} c_{2} c_{3}^{2}\right) \\
& =4 I_{2}^{2}-4 D_{2}^{2}+48 I_{1} I_{3} .
\end{aligned}
$$

As a final step we must evaluate $\Theta$, which is defined as the coefficient of $l m n$ in the reciprocal system

$$
l \Sigma+m \Sigma^{\prime}+n \Sigma^{\prime \prime}=0 .
$$

The expressions for $\Sigma, \Sigma^{\prime}, \Sigma^{\prime \prime}$ were given by (8). The discriminant can be written at once in determinant form, as

$$
\begin{aligned}
& \text { (19) } 8 a_{1} a_{2} a_{3} b_{1} b_{2} b_{3} c_{1} c_{2} c_{3}\left|\begin{array}{ccc}
0 & l a_{3}+m b_{3}+n c_{3} & l a_{2}+m b_{2}+n c_{2} \\
a_{3}+m b_{3}+n c_{3} & 0 & l a_{1}+m b_{1}+n c_{1} \\
a_{2}+m b_{2}+n c_{2} & l a_{1}+m b_{1}+n c_{1} & 0
\end{array}\right| \\
& (20)=16 a_{1} a_{2} a_{3} b_{1} b_{2} b_{3} c_{1} c_{2} c_{3}\left(l a_{1}+m b_{1}+n c_{1}\right)\left(l c_{2}+m b_{2}+n c_{2}\right)\left(l a_{3}+m b_{3}+n c_{3}\right) .
\end{aligned}
$$

The coefficient of $l m n$ in (20) is seen to be

Hence

$$
16 a_{1} a_{2} a_{3} b_{1} b_{2} b_{3} c_{1} c_{2} c_{3}\left(\sum^{6} a_{1} b_{2} c_{3}\right)=16 I_{1} I_{3}
$$

$$
\Theta=16 I_{1} I_{3} \text {. }
$$

Substituting the values $(15),(18)$ and $(21)$ in (13), we have after reduction

$$
T=D_{1}^{4}-8 D_{1}^{2} I_{2}+16 D_{2}^{2} \text {. }
$$

Substituting the values of $M I$ and $T$ thus found, in the condition $T^{2}=64 M I$, we have the theorem

If the 3-point and the 3-line are such that a conic may be inscribed in the 3line und circumscribed about the 3-point, then will 


$$
\left(D_{1}^{4}-8 D_{1}^{2} I_{2}+16 D_{2}^{2}\right)^{2}-512 I_{3} D_{1}^{3}\left(I_{1} D_{1}-4 I_{2}\right)=0 . *
$$

Forming the dual of (23) we get a theorem which admits of an important application. The dual is

$$
\begin{aligned}
& {\left[D_{1}^{*}-8 D_{1}^{4}\left(D_{1}^{*} I_{2}-2 I_{1} D_{1} D_{2}+6 D_{2}^{2}\right)+16 D_{1}^{4} D_{2}^{2}\right]^{2}} \\
& \quad-512 D_{1}^{6}\left(D_{1}^{3} I_{3}-D_{2}^{3}+\frac{1}{2} I_{1} D_{1} D_{2}^{2}-1_{2} I_{2} D_{1}^{2} D_{2}\right)\left(I_{1} D_{1}^{3}-2 D_{1}^{*} D_{2}\right)=0,
\end{aligned}
$$

which reduces to

$$
\begin{aligned}
& D_{1}^{\prime \prime}\left[D_{1}\left(D_{1}^{4}+64 I_{2}^{2}+32 I_{1} D_{1} D_{2}-16 D_{1}^{2} I_{2}-64 D_{2}^{2}\right)\right. \\
&-\left.512 I_{3}\left(I_{1} D_{1}-2 D_{2}\right)\right]=0 .
\end{aligned}
$$

Fron this we have the dual theorem, neglecting the trivial case where the three points are on a line:

$A$ conic may be dranon on the meets of the 3-line and touching the joins of the 3-point, if

$$
D_{1}\left(D_{1}^{4}+64 I_{2}^{2}+32 I_{1} D_{1} D_{2}-16 D_{1}^{2} I_{2}-6+D_{2}^{2}\right)-512 I_{3}\left(I_{1} D_{1}-2 D_{2}\right)=0 .
$$

Next consider equations (24) and (26) when two points of the 3-point are taken at the circular points at infinity, and the third point as a variable point $x$. They become equations of curves of the 8 th and 5 th degrees respectively. We shall discuss these curves briefly, as many properties can be deduced directly. $N^{T}$ will designate them by $R$ and $Q$ respectively. The equations of these curves can be written down explicitly by replacing $b_{i}$ and $c_{i}$ by the coördinates of $I$ and $J$ and $a_{i}$ by $x_{i}$.

In the case of conics inscribed in the 3-line and on the 3-point, if two of the points are taken at $I$ and $J$, all the conics become circles touching the 3-line. In other words; the octavic, $R=0$, is the locus of points from which circles can be drawn touching the lines of the 3-line.

In other words, $R=0$ is simply the equation of the 4 circles which can be drawn touching the 3 lines.

In the dual case, the join of $I$ and $J$, that is, the line at infinity, becomes a tangent to all the conics. Hence they are parabolas. Further the lines joining $I$ and $J$ to $x$ are to be tangents to the conics, so that $x$ is in every case a focus. Hence the theorem :

The locus of the foci of parabolas on three points is a quintic curve, and if the points are taken as vertices of the reference triangle, the equation of this quintic is

$$
Q=0 \text {. }
$$

It is possible to deduce many properties of $R$ and $Q$ without writing out the

* This was further verified by expanding SALMoN's alternative form, Conic Sections, p. 365. 
explicit equations. As a first step it is well to note what curves are represented by equating certain simple invariants to zero, when the points are taken as above.

$I_{3}=0$, is the equation of the reference triangle.

$D_{1}=0$, is the line at infinity.

$I_{1}=0$, is the polar line of $I$ and $J$ as to the 3 -line.

$D_{2}=0$, is the circumcircle.

$I_{1} D_{1}-2 D_{2}=0$, is the apolar circle.

$I_{1} D_{1}-4 D_{2}=0$, is the Feuerbach circle.

None of these require proof here except possibly the last. This can be shown directly from Hun's form, for he shows * that $N=0$ is the equation of the Fenerbach circle, and we have found $N^{\top}=I_{1} D_{1}-4 D_{2}$. Or it can be proved directly from the easily established fact that $I_{1} D_{1}-4 D_{2}=0$ is the locus of centers of rectangular hyperbolas on the vertices of the 3-line. But the feet of the three perpendiculars of the 3-line are clearly such centers and are also on the Feuerbach circle. Hence since $I_{1} D_{1}-4 D_{2}=0$ is a circle, it must be the Feuerbach circle. (All the conics $I_{1} D_{1}+\lambda D_{2}=0$ are circles, being on $I$ and $J$, the intersections of $D_{1}=0$ and $D_{2}=0$ ).

Now take the equation

$$
R=\left(D_{1}^{4}-8 D_{1}^{2} I_{2}+16 D_{2}^{2}\right)^{2}-512 I_{3} D_{1}^{3}\left(I_{1} D_{1}-4 D_{2}\right)=0,
$$

which we know to be the equation of the four touching circles.

If $I_{3}=0,\left(D_{1}^{4}-8 D_{1}^{2} I_{2}+16 D_{2}^{2}\right)^{2}=0$. Hence, the reference lines touch the octavic where they cut $K$, the quartic, $D_{1}^{4}-8 D_{1}^{2} I_{2}+16 D_{2}^{2}=0$.

If $I_{1} D_{1}-4 D_{2}=0, K^{2}=0$. Hence, the Feuerbach circle touches the octavic (or passes through double points) where it cuts $K$. This is really the well known theorem that the Feuerbach circle touches the inscribed and escribed circles.

The quartic $K$ is interesting in itself. From its equation,

$$
K=D_{1}^{4}-8 D_{1}^{2} I_{2}+16 D_{2}^{2}=0,
$$

we see that if $D_{1}=0, D_{2}^{2}=0$, and if $D_{2}=0$, either $D_{1}^{2}=0$ or $D_{1}^{2}-8 I_{2}=0$. This shows that $K$ is a bicircular quartic, that is, it has double points at $I$ and $J$. Since the latter are quadruple points of $R$, all the intersections of $K$ and $R$ are accounted for and easily constructed. There are 12 at the points where the reference triangle touches the $R$ circles, 4 where the Feuerbach circle touches them, and 8 at each of the circular points at infinity.

Since $R=0$ can be easily constructed, we will now take up $Q=0$. We can abbreviate

$$
Q=D_{1}\left[\left(D_{1}^{2}-8 I_{2}\right)^{2}+32 I_{1} D_{1} D_{2}-64 D_{2}^{2}\right]-512 I_{3}\left(I_{1} D_{1}-2 D_{2}\right)=0
$$

\footnotetext{
*'These Transactions, vol. 5 (1904), p. 47.
} 
by calling

therefore

$$
\begin{gathered}
D_{1}^{2}-8 I_{2}=U, \quad 16\left(I_{1} D_{1}-2 D_{2}\right)=V, \\
Q=D_{1}\left(U^{2}-D_{2} V\right)-32 I_{3} V=0 .
\end{gathered}
$$

In this if $D_{1}=0, I_{3} V=0$. That is, $Q$ cuts the line at infinity where $I_{3} V=0$ cuts it. But $I_{3}=0$ is the reference 3 -line and $V=0$ the apolar circle. Hence

The quintic $Q=0$, passes through the circular points at infinity and has its asymptotes parallel to the sides of the reference 3-line.

Again if $V=0, D_{1} U^{2}=0$. This says that the apolar circle touches the quintic (or cuts it at double points) where $U$ and $V$ intersect. Since $Q$ can be proven rational,* its six double points will not in general lie on a conic. Hence $V$ does not cut $Q$ in double points and we have the theorem :

The apolar circle, $V=0$, has fourfold contact with the quintic, $Q=0$, touching it at the four points where $U=0$ cuts $V=0$.

This theorem can be proven more directly by taking the dual of $V=0$, which is $I_{1} D_{1}-4 D_{2}=0$, or the Feuerbach circle, $F$. But, by the wellknown theorem, the Feuerbach circle touches each of the four tangent circles, $R$. Since contact is not destroyed in taking duals, $V$, the dual of $F$ touches $Q$, the dual of $R$ in four points which was the theorem to be verified. Rewrite $Q$, thus:

$$
Q=D_{1} U^{2}+2 V\left(D_{1} D_{2}-16 I_{3}\right)=D_{1} U^{2}-2 V W=0
$$

The curve $W=0$ is a cubic on the vertices of the reference triangle, cutting the line at infinity where the sides of the reference triangle meet it. That is, its asymptotes are parallel to sides of reference triangle.

When $W=0, U^{2}=0$ or $D_{1}=0$, hence $W=0$ touches $Q$ where $U$ cuts $W$.

But since $W$ and $Q$ have parallel asymptotes, it follows that three of the contacts are at infinity.

We can also find where $Q$ cuts the circumcircle. In $Q$, let $D_{2}=0$, and it becomes

$$
D_{1}\left(D_{1}^{2}-8 I_{2}\right)^{2}-512 I_{1} I_{3} D_{1}=0 .
$$

So unless the 3-point degenerates

$$
\left(D_{1}^{2}-8 I_{2}\right)^{2}-512 I_{1} I_{3}=0 \text {. }
$$

Then let $D_{2}=0$ in $R$ and drop $D_{1}^{4}$ as a factor, the result,

$$
\left(D_{1}^{2}-8 I_{2}\right)^{2}-512 I_{1} I_{3}=0 \text { : }
$$

*R. F. Davis, Educational Times, December 2, 1907, p. 545. 
is the same as (31). Hence:

$Q$ and $R$ cut the circumcircle in the same points.

It should be noted in this connection that the question, whether a locus is on any of the 6 fundamental points - that is, the points of the 3-point and the meets of the 3-line - is equivalent to the question whether the corresponding invariant vanishes, when there is a coincidence among the fundamental points. This is a question of some importance, but will not be discussed in detail here.

\section{§7. The Clebschians and their invariants.}

In the case of two general line cubics $(a \xi)^{3}=0$ and $(b \xi)^{3}=0$, the Clebschian is a point cubic defined by

$$
\chi=|a b x|^{3}=0 .
$$

Similarly the Clebschian of two point cubics $(\alpha x)^{3}=0$ and $(\beta x)^{3}=0$ is defined by

$$
\chi^{\prime}=|\alpha \beta \xi|^{3}=0 \text {. }
$$

If, as in the case of two triangles, the curves are of both the third class and third order, the two Clebschians are in general different. In forming the Clebschians both triangles or cubics must be taken in points or $b^{-t h}$ in lines. So the work here is slightly different from the preceding sections.

If one cubic is taken as the reference 3-point and the other as $(a \xi)^{3}=0$, from (1) we have

$$
\chi=6\left(a_{2} a_{3}^{2} x_{1}^{2} x_{2}+a_{1} a_{2}^{2} x_{1} x_{3}^{2}+a_{1}^{2} a_{3} x_{2}^{2} x_{3}-a_{1} a_{3}^{2} x_{1} x_{2}^{2}-a_{1}^{2} a_{2} x_{2} x_{3}^{2}-a_{2}^{2} a_{3} x_{1}^{2} x_{3}\right)
$$

If in turn the second cubic is taken as the 3-point $(a \xi)(b \xi)(c \xi)=0$ we must replace

and this gives us

$$
3 a_{k} a_{l}^{2} \text { by }\left(a_{k} b_{l} c_{l}+a_{l} b_{k} c_{l}+a_{l} b_{l} c_{k}\right) \text {, }
$$

$$
\begin{aligned}
\chi=2 & {\left[\left(a_{2} b_{3} c_{3}+a_{3} b_{2} c_{3}+a_{3} b_{3} c_{2}\right) x_{1}^{2} x_{2}+\left(a_{1} b_{2} c_{2}+a_{2} b_{1} c_{2}+a_{2} b_{2} c_{1}\right) x_{1} x_{3}^{2}\right.} \\
& +\left(a_{3} b_{1} c_{1}+a_{1} b_{3} c_{1}+a_{1} b_{1} c_{3}\right) x_{2}^{2} x_{3}-\left(a_{1} b_{3} c_{3}+a_{3} b_{1} c_{3}+a_{3} b_{3} c_{1}\right) x_{1} x_{2}^{2} \\
& \left.-\left(a_{2} b_{1} c_{1}+a_{1} b_{2} c_{1}+a_{1} b_{1} c_{2}\right) x_{2} x_{3}^{2}-\left(a_{3} b_{2} c_{2}+a_{2} b_{3} c_{2}+a_{2} b_{2} c_{3}\right) x_{1}^{2} x_{3}\right] .
\end{aligned}
$$

It will aid in clearness to define $\chi$ geometrically. If we call the reference 3 -point $d, e, f$, then $\chi$ may be defined as the locus of points $y$ such that the two triads of lines $y-d, e, f$ and $y-a, b, c$ are apolar. $\chi^{\prime}$ is the locus of lines $\eta$ having the dual property. From (4), we see that $\chi$ is on the vertices of the reference 3-point, and, since the two 3-points enter symmetrically, we have the theorem : 
The Clcbschian of two 3-points is on all six points.*

Further since (4) contains no terms in $x_{1} x_{2} x_{3}$, the reference triangle is apolar to it. Hence,

The Clebschian of two 3-points is a cubic to which both 3-points are apolar. The dual theorems for two 3-lines can be written down at once.

Since the invariants, $S$ and $T$, of $\chi$ are mutual invariants of the two 3 points, they must be expressible in terms of our fundamental invariants.

For $S$, we will make use of SaLMON's standard form. $\dagger$ In $\chi$, the SALMON coefficients have the following equivalents,

$$
\begin{array}{cc}
a=b=c=m=0, & a_{2}=\left(a_{2} b_{3} c_{3}+a_{3} b_{2} c_{3}+a_{3} b_{3} c_{2}\right), \\
a_{3}=-\left(a_{3} b_{2} c_{2}+a_{2} b_{3} c_{2}+a_{2} b_{2} c_{3}\right), & b_{1}=-\left(a_{1} b_{3} c_{3}+a_{3} b_{1} c_{3}+a_{3} b_{3} c_{1}\right), \\
b_{3}=\left(a_{3} b_{1} c_{1}+a_{1} b_{3} c_{1}+a_{1} b_{1} c_{3}\right), & c_{1}=-\left(a_{2} b_{1} c_{1}+a_{1} b_{2} c_{1}+a_{1} b_{1} c_{2}\right), \\
c_{2}=\left(a_{1} b_{2} c_{2}+a_{2} b_{1} c_{2}+a_{2} b_{2} c_{1}\right) .
\end{array}
$$

The nonessential, common numerical factor has been dropped. This gives, by substituting in SALMon's form,

$$
\begin{aligned}
S= & -\left(a_{1} b_{3} c_{3}+a_{3} b_{1} c_{3}+a_{3} b_{3} c_{1}\right)^{2}\left(a_{1} b_{2} c_{2}+a_{2} b_{1} c_{2}+a_{2} b_{2} c_{1}\right)^{2} \\
& -\left(a_{2} b_{3} c_{3}+a_{3} b_{2} c_{3}+a_{3} b_{3} c_{2}\right)^{2}\left(a_{2} b_{1} c_{1}+a_{1} b_{2} c_{1}+a_{1} b_{1} c_{2}\right)^{2} \\
& -\left(a_{3} b_{2} c_{2}+a_{2} b_{3} c_{2}+a_{2} b_{2} c_{3}\right)^{2}\left(a_{3} b_{1} c_{1}+a_{1} b_{3} c_{1}+a_{1} b_{1} c_{3}\right)^{2} \\
& +\left(a_{2} b_{3} c_{3}+a_{3} b_{2} c_{3}+a_{3} b_{3} c_{2}\right)\left(a_{2} b_{1} c_{1}+a_{1} b_{2} c_{1}+a_{1} b_{1} c_{2}\right) \\
& \left(a_{3} b_{2} c_{2}+a_{2} b_{3} c_{2}+a_{2} b_{2} c_{3}\right)\left(a_{3} b_{1} c_{1}+a_{1} b_{3} c_{1}+a_{1} b_{1} c_{3}\right) \\
& +\left(a_{3} b_{2} c_{2}+a_{2} b_{3} c_{2}+a_{2} b_{2} c_{3}\right)\left(a_{3} b_{1} c_{1}+a_{1} b_{3} c_{1}+a_{1} b_{1} c_{3}\right) \\
& \left(a_{1} b_{3} c_{3}+a_{3} b_{1} c_{3}+a_{3} b_{3} c_{1}\right)\left(a_{1} b_{2} c_{2}+a_{2} b_{1} c_{2}+a_{2} b_{2} c_{1}\right) \\
& +\left(a_{1} b_{3} c_{3}+a_{3} b_{1} c_{3}+a_{3} b_{3} c_{1}\right)\left(a_{1} b_{2} c_{2}+a_{2} b_{1} c_{2}+a_{2} b_{2} c_{1}\right) \\
& \left(a_{2} b_{1} c_{1}+a_{1} b_{2} c_{1}+a_{1} b_{1} c_{2}\right)\left(a_{2} b_{3} c_{3}+a_{3} b_{2} c_{3}+a_{3} b_{3} c_{2}\right) .
\end{aligned}
$$

Expanding and collecting the above we get

(6) $S=16\left[\sum^{18} a_{1}^{3} a_{2} b_{1} b_{2}^{2} b_{3} c_{2} c_{3}^{3}-\sum^{9} a_{1}^{2} a_{2}^{2} b_{1} b_{2} b_{3}^{2} c_{1} c_{2} c_{3}^{2}-\sum^{9} a_{1}^{4} b_{2}^{2} b_{3}^{2} c_{2}^{2} c_{3}^{2}\right]$,

or using the symbols of $\S 3$,

$$
S=16(N-H-K) .
$$

* These two theorems lay no claim to uovelty, see F. MORLEY, these Transactious, vol. 5 (1!04), p. 472, for a discussion.

† ITikere Ebene Kurven, p. 247. 
Employing the fact that the $D$ 's must enter in each term to an even power, if at all, we find that the only combinations entering are

$$
\begin{aligned}
I_{2}\left(D_{1}^{2}+I_{1}^{2}\right) & =2 N+4 E+10 F+2 G+8 H, \\
I_{2}^{2}-D_{2}^{2} & =4 H, \\
\left(I_{1}^{2}-D_{1}^{2}\right)^{2} & =32 N+64 H+16 K, \\
I_{1} D_{1} D_{2} & =-N+2 E+5 F+G-4 H .
\end{aligned}
$$

It is not difficult to select the proper coefficients ; the result is

$$
\begin{gathered}
S=12 I_{2}\left(D_{1}^{2}+I_{1}^{2}\right)-36\left(I_{2}^{2}-D_{2}^{2}\right)-\left(I_{1}^{2}-D_{1}^{2}\right)^{2}-24 I_{1} D_{1} D_{2} \\
=4\left(I_{1} D_{1}-3 D_{2}\right)^{2}-\left(I_{1}^{2}+D_{1}^{2}-6 I_{2}\right)^{2} .
\end{gathered}
$$

Geometrically $S=0$ is the condition that the Hessian of the Clebschian of the two 3-points shall be three lines.

Since the Clebschian treats the two triangles symmetrically, it is clear that $S$ should be self dual. Applying the formulæ of $\S 3$, we get

$$
S^{\prime}=D_{1}^{4} S^{\prime}
$$

This is a good check on the work.

To substitute the coefficients of $\chi$ directly in SaLmon's form for $T^{*}$ leads to complicated expressions. The following method presents fewer difficulties.

Let $(\alpha x)^{3}$ and $\left(a \xi j^{3}\right.$ be two cubics giving the connex

$$
(\alpha a)^{2}(\alpha x)(a \xi)=0 \text {. }
$$

The 3 fixed points of the connex are given by

$$
a_{i}(\alpha x)(\alpha a)^{2}=\lambda x_{i} ;
$$$$
(i=1,2,3) \text {. }
$$

If the 3 equations of (12) are consistent

$$
\Delta(\lambda)=\left|\begin{array}{lll}
a_{1} \alpha_{1}(\alpha a)^{2}+\lambda & a_{2} \alpha_{1}(\alpha a)^{2} & a_{3} \alpha_{1}(\alpha u)^{2} \\
a_{1} \alpha_{2}(\alpha a)^{2} & a_{2} \alpha_{2}(\alpha a)^{2}+\lambda & a_{3} \alpha_{2}(\alpha a)^{2} \\
a_{1} \alpha_{3}(\alpha a)^{2} & a_{2} \alpha_{3}(\alpha a)^{2} & a_{3} \alpha_{3}(\alpha a)^{2}+\lambda
\end{array}\right| \equiv 0
$$

The coefficients of powers of $\lambda$ in $\Delta(\lambda)$ are invariants of the connex. Now let $(\alpha x)^{3}$ become the reference 3-line and

$$
\Delta(\lambda)=\left|\begin{array}{ccc}
2 a_{1} a_{2} a_{3}+\lambda & 2 a_{2}^{2} a_{3} & 2 a_{2} a_{3}^{2} \\
2 a_{1}^{2} a_{3} & 2 a_{1} a_{2} a_{3}+\lambda & 2 a_{1} a_{3}^{2} \\
2 a_{1}^{2} a_{2} & 2 a_{2}^{2} a_{1} & 2 a_{1} a_{2} a_{3}+\lambda
\end{array}\right|
$$

\footnotetext{
* Höhere Ebene Kurven, p. 248.
} 
If the second cubic is taken as

$$
\begin{aligned}
a \xi_{1}^{3}+b \xi_{2}^{3}+c \xi_{3}^{3}+3 a_{2} \xi_{1}^{2} \xi_{2} & +3 a_{3} \xi_{1}^{2} \xi_{3}+3 b_{1} \xi_{2}^{2} \xi_{1} \\
& +3 b_{3} \xi_{2}^{2} \xi_{3}+3 c_{1} \xi_{3}^{2} \xi_{1}+3 c_{2} \xi_{3} \xi_{2}+6 m \xi_{1} \xi_{2} \xi_{3}=0
\end{aligned}
$$

$\Delta(\lambda)$ takes the form, dropping numerical factors,

where

$$
\Delta(\lambda)=\left|\begin{array}{ccc}
m+\lambda & a_{3} & a_{2} \\
b_{3} & m+\lambda & b_{1} \\
c_{2} & c_{1} & m+\lambda
\end{array}\right|=\lambda^{3}+J_{1} \lambda^{2}+J_{2} \lambda+J_{3},
$$

$$
\begin{aligned}
& J_{1}=3 m=3 I_{1}, \\
& J_{2}=3 m^{2}-\left(b_{1} c_{1}+c_{2} a_{2}+a_{3} b_{3}\right), \\
& J_{3}=\left|\begin{array}{lll}
m & a_{3} & a_{2} \\
b_{3} & m & b_{1} \\
c_{2} & c_{1} & m
\end{array}\right|
\end{aligned}
$$

Now the Clebschian of the reference 3-line and the general cubic is

$$
\chi=c_{2} x_{1}^{2} x_{2}+b_{1} x_{1} x_{3}^{2}+a_{3} x_{2}^{2} x_{3}-c_{1} x_{1} x_{2}^{2}-a_{2} x_{3}^{2} x_{2}-b_{3} x_{1}^{2} x_{3}=0,
$$

and $T$ for this is

$$
\begin{aligned}
T= & 6 b_{1} c_{1} c_{2} a_{2} a_{3} b_{3}-8\left(b_{1}^{3} c_{1}^{3}+c_{2}^{3} a_{2}^{3}+a_{3}^{3} b_{3}^{3}\right)-27\left(a_{3}^{2} b_{1}^{2} c_{2}^{2}+a_{2}^{2} b_{3}^{2} c_{1}^{2}\right) \\
& +12\left(b_{1}^{2} c_{1}^{2} a_{2} c_{2}+b_{1}^{2} c_{1}^{2} a_{3} b_{3}+c_{2}^{2} a_{2}^{2} a_{3} b_{3}+c_{2}^{2} a_{2}^{2} b_{1} c_{1}+a_{3}^{2} b_{3}^{2} b_{1} c_{1}+a_{3}^{2} b_{3}^{2} c_{2} a_{2}\right) .
\end{aligned}
$$

Or we can write

$$
T=6 x-8 y-27 z+12 t .
$$

From the expressions derived above we have

$$
\begin{gathered}
\left(3 I_{1}^{2}-J_{2}\right)^{3}=\sum^{3} b_{1}^{3} c_{1}^{3}+3 \sum^{6} b_{1}^{2} c_{1}^{2} a_{2} c_{2}+6 b_{1} c_{1} c_{2} a_{2} a_{3} b_{3}=6 x+y+3 t \\
\left(J_{3}+2 I_{1}^{3}-I_{1} J_{2}\right)^{2}=a_{2}^{2} b_{3}^{2} c_{1}^{2}+a_{3}^{2} b_{1}^{2} c_{2}^{2}+2 b_{1} c_{1} c_{2} a_{2} a_{3} b_{3}=2 x+z, \\
S\left(3 I_{1}^{3}-J_{2}\right)=-\sum^{3} b_{1}^{3} c_{1}^{3}+3 b_{1} c_{1} c_{2} a_{2} a_{3} b_{3}=3 x+y .
\end{gathered}
$$

Elimination of $x, y, z, t$ from these four equations gives

$$
T=4\left(3 I_{1}^{2}-J_{2}\right)^{3}-27\left(J_{3}+2 I_{1}^{3}-I_{1} J_{2}\right)^{2}+12 S\left(3 I_{1}^{2}-J_{2}\right) \text {. }
$$

But $I_{1}$ is identical with the $I_{1}$ of the present system, $J_{2}$ and $J_{3}$ are $I_{2}$ and $-I_{3}$ of Hun's system, and $S$ is given by (9). 
Substituting these values in (21) we find

$$
\begin{aligned}
T=-8\left[\left(I_{1}^{2}-D_{1}^{2}\right)^{3}-216 I_{2}^{3}-\right. & 18 I_{2}\left(I_{1}^{4}-D_{1}^{4}\right) \\
+ & 108\left(I_{1}^{2} I_{2}^{2}+D_{1}^{2} D_{2}^{2}\right)-54\left(D_{1} I_{2}-I_{1} D_{2}\right)^{2} \\
+ & \left.36 I_{1} D_{1} D_{2}\left(I_{1}^{2}-D_{1}^{2}\right)-648 I_{2} D_{2}^{2}\right] .
\end{aligned}
$$

Applying the dual formulas we have $T^{\prime}=D_{1}^{6} T$, again a check on the work.

As a further example, the invariant condition on the two triangles, that the two Clebschians may be apolar, will be calculated. The outline, only, of the work shall be given. The form of $\chi$ is given in full by equation (4). To get the equation of $\chi$, the coefficients of the corresponding terms can be derived from $\chi$ by replacing each letter by its minor in $D_{1}$. Call the minors $A_{1}, B_{1}$, etc., as in $\S 3$. Then

$$
\begin{aligned}
\chi^{\prime}= & \left(A_{2} B_{3} C_{3}+A_{3} B_{2} C_{3}+A_{3} B_{3} C_{2}\right) \xi_{1} \xi_{2}+\left(A_{1} B_{2} C_{2}+A_{2} B_{1} C_{2}+A_{2} B_{2} C_{1}\right) \xi_{1} \xi_{3}^{2} \\
& +\left(A_{3} B_{1} C_{1}+A_{1} B_{3} C_{1}+A_{1} B_{1} C_{3}\right) \xi_{2}^{2} \xi_{3}-\left(A_{1} B_{3} C_{3}+A_{3} B_{1} C_{3}+A_{3} B_{3} C_{1}\right) \xi_{1} \xi_{2}^{2} \\
& -\left(A_{2} B_{1} C_{1}+A_{1} B_{2} C_{1}+A_{1} B_{1} C_{2}\right) \xi_{2} \xi_{3}^{2}-\left(A_{3} B_{2} C_{2}+A_{2} B_{3} C_{2}+A_{2} B_{2} C_{3}\right) \xi_{1}^{2} \xi_{3}
\end{aligned}
$$

To find the apolarity condition, regard, as usual, the $\xi$ 's as differential operators and equate the result to zero. This gives

$$
\begin{aligned}
& \left(a_{2} b_{3} c_{3}+a_{3} b_{2} c_{3}+a_{3} b_{3} c_{2}\right)\left(A_{2} B_{3} C_{3}+A_{3} B_{2} C_{3}+A_{3} B_{3} C_{2}\right) \\
& \quad+\left(a_{1} b_{2} c_{2}+a_{2} b_{1} c_{2}+a_{2} b_{2} c_{1}\right)\left(A_{1} B_{2} C_{2}+A_{2} B_{1} C_{2}+A_{2} B_{2} C_{1}\right) \\
& +\left(a_{3} b_{1} c_{1}+a_{1} b_{3} c_{1}+a_{1} b_{1} c_{3}\right)\left(A_{3} B_{1} C_{1}+A_{1} B_{3} C_{1}+A_{1} B_{1} C_{3}\right) \\
& +\left(a_{1} b_{3} c_{3}+a_{3} b_{1} c_{3}+a_{3} b_{3} c_{1}\right)\left(A_{1} B_{3} C_{3}+A_{3} B_{1} C_{3}+A_{3} B_{3} C_{1}\right) \\
& +\left(a_{2} b_{1} c_{1}+a_{1} b_{2} c_{1}+a_{1} b_{1} c_{2}\right)\left(A_{2} B_{1} C_{1}+A_{1} B_{2} C_{1}+A_{1} B_{1} C_{2}\right) \\
& +\left(a_{3} b_{2} c_{2}+a_{3} b_{3} c_{2}+a_{2} b_{2} c_{3}\right)\left(A_{3} B_{2} C_{2}+A_{2} B_{3} C_{2}+A_{2} B_{2} C_{3}\right)=0 .
\end{aligned}
$$

By replacing the capitals by minors in the small letters and expanding, the whole can be summed thus :

$$
\begin{aligned}
2\left(\sum^{8} a_{1} a_{2} a_{3} b_{2}^{2} b_{3} c_{1}^{2} c_{3}-\sum^{\circ} a_{1} a_{2} a_{3} b_{1}^{2} b_{3} c_{2}^{2} c_{3}\right) & +3\left(\sum^{6} a_{1} a_{2}^{2} b_{2} b_{3}^{2} c_{3} c_{1}^{2}-\sum^{6} a_{1} a_{2}^{2} b_{3} b_{1}^{2} c_{2} c_{3}^{2}\right) \\
& +2\left(\sum^{9} a_{1}^{3} b_{2} b_{3}^{2} c_{2}^{2} c_{3}-\sum^{8} a_{1}^{3} b_{2}^{2} b_{3} c_{2} c_{3}^{2}\right)=0 .
\end{aligned}
$$

Expressed in terms of the fundamental invariants, this is

$$
D_{1}^{3}-I_{1}^{2} D_{1}-3 I_{1} D_{2}+9 I_{2} D_{1}=0 \text {. }
$$


Hence: The two Clebschians of two triangles are apolar if

$$
D_{1}^{3}-I_{1}^{2} D_{1}-3 I_{1} D_{2}+9 I_{2} D_{1}=0 \text {. }
$$

Of necessity, this form is self-dual.

The invariants of $\chi^{\prime}$ are seen to be the dual of those of $\chi$. But $S=S$ and $T^{\prime}=T$. Hence: If neither triangle is degenerate, $S=0$ is the condition that the Hessian of one Clebschian break down into 3 lines and at the same time that the Hessian of the other break down into 3 points.

There are a number of other interesting cubic covariants and contravariants of two triangles. But the limits of this paper will not permit of a detailed treatment, the above discussion of the Clebschian being itself meager and incomplete.

\section{§8. Self-dual invariant forms.}

A number of self-dual forms have appeared in the course of this article, and the question naturally arises as to the number of such self-dual forms which are independent. Such forms are of special importance, because the vanishing of one of them indicates a mutual relation between the two triangles. The most general self-dual invariant of given degree can be found by carrying out the dual transformation on the most general invariant of that degree, and asking that the invariant be left unaltered save for multiplicative powers of $D_{1}$. For example, suppose the most general self-dual invariant of degree 3 is required. We ask that

$$
\begin{aligned}
& D_{1}^{3}\left(a I_{1}^{3}+b D_{1}^{3}+c I_{1}^{2} D_{1}+d I_{1} D_{1}^{2}+e I_{1} I_{2}+f I_{1} D_{2}+g D_{1} D_{2}+h I_{2} D_{1}+2 k I_{3}\right) \\
& \quad=a\left(I_{1}^{3} D_{1}^{3}-18 I_{1}^{2} D_{1}^{2} D_{2}+108 I_{1} D_{1} D_{2}^{2}-216 D_{2}^{3}\right)+b D_{1}^{6} \\
&+c D_{1}^{2}\left(I_{1}^{2} D_{1}^{2}-12 I_{1} D_{1} D_{2}+36 D_{2}^{2}\right)+d\left(I_{1} D_{1}^{3}-6 D_{1}^{4} D_{2}\right) \\
&+e\left(I_{1} I_{2} D_{1}^{3}-2 I_{1}^{2} D_{1}^{2} D_{2}+18 I_{1} D_{1} D_{2}^{2}-6 I_{2} D_{1}^{2} D_{2}-36 D_{2}^{3}\right) \\
&++f\left(-I_{1} D_{1}^{3} D_{2}+6 D_{1}^{2} D_{2}^{2}\right)-g\left(D_{1}^{4} D_{2}\right)+h\left(D_{1}^{4} I_{2}-2 I_{1} D_{1}^{3} D_{2}+6 D_{1}^{2} D_{2}^{2}\right) \\
&+k\left(2 D_{1}^{3} I_{3}-2 D_{2}^{3}+I_{1} D_{1} D_{2}^{2}-I_{2} D_{1}^{2} D_{2}\right)
\end{aligned}
$$

Equating coefficients, we get the equalities $\mathrm{c}=-9 a, k=54 a, 6 c=-(f+h)$, $g=-3 d$. Introducing these in the left of $(1)$, we have as the most general self-dual form of degree 3 ,

(8) $a\left(I_{1}^{3}-9 I_{1} I_{2}+108 I_{3}\right)+d\left(D_{1}^{3}-3 D_{1} D_{2}\right)+c\left(I_{1}^{2} D_{1}-6 I_{1} D_{2}\right)+h\left(I_{2} D_{1}-I_{1} D_{2}\right)$. 
Since $a, c, d$ and $h$ are arbitrary constants it follows that the expressions in parentheses are all self-dual forms, as can be readily verified. In the further study of self-dual forms, we get the following system of five independent ones, which form a complete system :
(1) $D_{1}$,
(2) $I_{1} D_{1}-3 D_{2}$,
(3) $I_{1}^{2}-6 I_{2}$,
(4) $I_{2} D_{1}-I_{1} D_{2}$
(5) $I_{1}^{3}-9 I_{1} I_{2}+108 I_{3}$.

To prove this system complete let us designate forms (1), (2), (3), (4), (5) by $A, B, C, E$, and $F$ respectively. We then have the following equations :

$$
\begin{aligned}
& D_{1}=A, \\
& D_{2}=-\frac{1}{3}\left(B-I_{1} A\right)=\frac{1}{3}\left(A I_{1}-B\right), \\
& I_{2}=\frac{1}{6}\left(I_{1}^{2}-C\right), \\
& I_{3}=\frac{1}{108}\left(F-I_{1} C+3 I_{1} I_{2}\right)=\frac{1}{2} \frac{1}{16}\left(F-3 C I_{1}+I_{1}^{3}\right) .
\end{aligned}
$$

That is, $D_{1}, D_{2}, I_{2}$, and $I_{3}$ can each be expressed in terms of $I_{1}$ and self-dual forms.

From (4) or $E$ we get the further equation

$$
E=\frac{A}{6}\left(I_{1}^{2}-C\right)-\frac{I_{1}}{3}\left(A I_{1}-B\right) \quad \text { or } \quad 6 E=2 B I_{1}-A C-A I_{1}^{2},
$$

and hence

$$
I_{1}^{2}=\frac{6 E+A C-2 B I_{1}}{A},
$$

a formula which enables us to reduce higher powers of $I_{1}$. Hence from this series of equations we see that any invariant can be expressed as a rational function of the five self-dual forms $A, B, C, E, F$, and of $I_{1}$ to the first degree. That is any invariant $I$ can be written as

$$
I=S_{1}+k I_{1} S_{2}
$$

where $S_{1}$ and $S_{2}$ are rational functions of $A, B, C, E$, and $F$. Now suppose $I$ is self-dual and written in form (7). Since $S_{1}$ and $S_{2}$ have been proven self-dual, it follows that (7) can be true only for $k I_{1} S_{2}=0$, as it can obviously not be self-dual, $I_{1}$ not being self-dual. Therefore if self-dual $I=S_{1}$, that is, a rational function of $A, B, C, E, F$. This proves the theorem.

Any form which can be expressed in terms of these five is self-dual, and it is frequently easier to show the self-duality of a form by expressing it in terms of 
$A, B, C, E, F$, than by applying the dual transformation formulæ. Only the first two of the five have a simple geometrical interpretation known to the writer.

\section{\$9. The fundamental invariants under a special Cremona transformation.}

It is the purpose here to show the effect of a special Cremona transformation on the fundamental invariants, and to apply the result to a single example.

Take the simple, quadratic involutory transformation

$$
y_{i}=\frac{k}{x_{i}}
$$

The effect of this transformation on the invariants is to replace each symbol by its reciprocal. Since all invariant forms are homogeneous, $k$ will appear to the same power in each term, hence may be taken as unity without loss of generality. The fundamental invariants, under this transformation, become

$$
\begin{gathered}
D_{1}^{\prime}=\frac{D_{2}}{I_{3}}, \quad I_{1}^{\prime}=\frac{I_{2}}{I_{3}}, \\
D_{2}^{\prime}=\frac{D_{1}}{I_{3}}, \quad I_{2}^{\prime}=\frac{I_{1}}{I_{3}}, \quad I_{3}^{\prime}=\frac{1}{I_{3}} .
\end{gathered}
$$

As a typical illustration we take the rational quintic,

$$
x_{i}=\frac{t-\alpha_{i}}{\left(t-\beta_{i}\right)^{2}}
$$$$
(i=1,2,3)
$$

This quintic has 3 cusps, 3 double points, 3 double tangents, 3 flexes, and is of class 5. It follows from the simple counting of constants that any two of the singular triangles can not be taken arbitrarily, but have a single relation connecting them. We shall give in detail but a single one of these relations. Taking the triangle of double points as the reference 3-line, we ask: What is the condition on the triangle of cusps?

From the theory of Cremona transformations," it is well known that if the above involutory transformation is carried out on the given quintic, with the double points as fundamental points, the quintic goes into a rational quartic with 3 cusps, and with simple points at the 3 fundamental points. If we next carry out the same transformation regarding the cusps as fundamental points (which change of fundamental points is evidently equivalent to taking the dual), the rational quartic goes into a conic which touches the original fundamental lines, and has the three cusp points as ordinary points. But we already know

* Clafbsch-LindemanN, vol. I, pp. 478, fi., where a number of references are given. 
from an earlier paragraph the condition that a conic may be inscribed in the 3line and circumseribed about the 3-point. It is

$$
\left(D_{1}^{4}-8 D_{1}^{2} I_{2}+16 D_{2}^{2}\right)^{2}-512 I_{3} D_{1}^{3}\left(I_{1} D_{1}-4 D_{2}\right)=0 .
$$

Hence to tind the condition on the cusp triangle, we must take (2) and carry out the above transformations in reverse order. Being of period 2 the inverses are the same as the direct transformations. That is, transformation (1) must be carried out first, then the dual transformation, and finally (1) a second time. This gives an expression of the 24th degree in the coefficients of the original triangles.

If on the other hand the cusp triangle is taken as the reference 3-line the condition on the 3-point of double points turns out to be of the 18th degree by a similar argument. It is

$$
\begin{aligned}
& D_{1}^{s} D_{2}^{8}+16\left(I_{1}^{2} D_{1}^{2} D_{3}-4 I_{1} D_{1} D_{2}^{2}+4 D_{2}^{3}-\right.\left.D_{1}^{4} D_{2}-16 I_{3} D_{1}^{3}\right) \\
& \times\left(2 D_{1}^{3} I_{3}-2 D_{2}^{3}+I_{1} D_{1} D_{2}^{2}-I_{2} D_{2}^{2} D_{2}\right)^{2} \\
&-8 D_{2}\left(2 I_{2} D_{1}^{8} D_{2}-3 I_{1} D_{1}^{s} D_{2}^{2}+6 D_{1}^{4} D_{2}^{3}\right)\left(2 D_{1}^{3} I_{3}-2 D_{2}^{3}+I_{1} D_{1} D_{2}^{2}-I_{2} D_{1}^{2} D_{2}\right)=0 .
\end{aligned}
$$

This is sufficient to illustrate our point. For by direct attack this problem is practically impossible, yet by this transformation it becomes comparatively simple. Other examples can readily be found to further illustrate this idea.

Incidentally this gives a convenient geometrical interpretation for $I_{2}=0$, the only one of the fundamental invariants not previously interpreted. For if the transformation $y_{i}=1 / x_{i}$ is carried out on the three points of the 3-point, they are carried into three new points, whose equations are

$$
\begin{gathered}
a_{2} a_{3} \xi_{1}+a_{3} a_{1} \xi_{2}+a_{1} a_{2} \xi_{3}=0, \\
b_{2} b_{3} \xi_{1}+b_{3} b_{1} \xi_{2}+b_{1} b_{2} \xi_{3}=0, \\
c_{2} c_{3} \xi_{1}+c_{3} c_{1} \xi_{2}+c_{1} c_{2} \xi_{3}=0 .
\end{gathered}
$$

Clearly the condition that these shall be apolar to the reference 3 line is

$$
I_{2}=0 \text {. }
$$

Or as a definite theorem: If two triangles are such that when one is taken as $a$ 3-line and the other as a 3-point, and the transformation $y_{i}=1 / x_{i}$ is carried out, the 3-point is apolar to the 3-line, then will

$$
I_{2}=0 \text {. }
$$


This gives, however, no direct information as to the projective relation between the 3-line and 3-point in their original position. Other interpretations of $I_{2}=0$ arose, but none so direct and simple as for the vanishing of the other fundamental invariants.

JoHNS HOPKINS UNIVERSITY,

February, 1909. 